\title{
DEFORMATIONS OF INTEGRALS OF EXTERIOR DIFFERENTIAL SYSTEMS
}

BY

DOMINIC S. P. LEUNG ( $\left.{ }^{1}\right)$

\begin{abstract}
On any general solution of an exterior differential system $I, a$ system of linear differential equations, called the equations of variation of $I$, is defined. Let $\mathrm{v}$ be a vector field defined on a general solution of $I$ such that it satisfies the equations of variation and wherever it is defined, $v$ is either the zero vector or it is not tangential to the general solution. By means of some associated differential systems and the fundamental theorem of CartanKähler theory, it is proved that, under the assumption of real analyticity, $v$ is locally the deformation vector field of a one-parameter family of general solutions of $I$. As an application, it is proved that, under the assumption of real analyticity, every Jacobi field on a minimal submanifold of a Riemannian manifold is locally the deformation vector field of a one-parameter family of minimal subma nif olds.
\end{abstract}

0 . Introduction. The theory of analytic exterior differential systems was developed by E. Cartan for the study of infinite pseudo-groups. E. Kähler completed the theory and generalized it. It reduces the existence of solutions (called general) to a purely algebraic problem. Such a general solution depends on the initial data which in turn depends on arbitrary functions. So it is of interest to study a family of solutions of an exterior differential system, in particular, a one-parameter family of general solutions. For a compatible system $I$ of ordinary differential equations, the following facts are well known (see for example [2]). Along any solution $N$ of $I$, a system of linear differential equations, called the equations of variation of $I$, is defined. Any vector field $\mathbf{v}$ defined on $N$ which satisfies the equations of variation is the deformation vector field of a one-parameter family of solutions of $I$ on $N$. In particular, if $I$ is the system which defines geodesics on a Riemannian manifold, we have that every Jacobi field along a geodesic may be obtained by a variation through geodesics. The purpose of this paper is to generalize the above to an arbitrary general solution of an exterior differential system. We will prove the following (see the Main Theorem):

Received by the editors September 17, 1971.

AMS 1970 subject class ifications. Primary 58A15; Secondary 53A10, 53B25.

Key words and phrases. Exterior differential system, Cartan-Kähler the ory, general solution, regular integral chain, one-parameter family of integral manifolds, deformation vector, equations of variation, I-field, normal system, normal solution, normal data, associated differential systems, minimal subma nifolds, Jacobi field.

(1) This research was supported in part by NSF GP 9613. 
On any general solution $N$ of an exterior differential system $I$ on a manifold $M$, the equations of variation are defined. Let $\mathbf{v}$ be a vector field defined on $N$ which satisfies the equations of variation and such that for $m \in N, \mathbf{v}(m)$ is either the zero vector or it is not in $T_{m}(N)$. Then under the assumption of real analyticity $\mathbf{v}$ is locally the deformation vector field of a one-parameter family of integral manifolds of $I$ on $N$. As an application, we will prove that under the assumption of real analyticity every Jacobi field defined on a minimal submanifold can be obtained (locally) as the deformation vector field of a one-parameter family of minimal submanifolds. This solves the problem as posed in [12] in the real analytic case.

In $\$ 1$, we will give a brief summary of Cartan-Kähler theory of exterior differential systems. Regular integral elements and involutiveness will be defined in terms of the necessary and sufficient conditions for the existence of a regular integral chain as proved in $[7, \mathrm{p} .40]$. This approach may not be the most natural, but it immediately reduces the problem of finding general solutions essentially to that of checking the compatibility and ranks of systems of linear equations. In $\S \S 2-5$, the Main Theorem will be formulated and proved. We will begin by establishing that it is sufficient to consider a class of Pfaffian systems called normal systems. Associated with a normal system, two other differential systems are defined. By means of the fundamental the orem of Cartan-Kähler theory, they will be used to construct the required one-parameter family of integral manifolds and also to prove that its deformation vector field coincides with the given vector field. In $\$ 6$, after some computations we will apply the Main The orem to Jacobi fields defined on minimal submanifolds of a Riemannian manifold.

This paper is a continuation of research done at University of California, in a doctoral thesis under the direction of Professor S. S. Chern. I wish to thank him for his advice and encouragement.

Throughout this paper all functions, manifolds, submanifolds and associated differential geometric structures will be assumed to be real analytic. When no confusion is likely, we will simply regard an immersed submanifold of a manifold $M$ defined by $f: N \rightarrow M$ as a subset $f(N)$ of $M$ and its tangent space $T_{x}(N)$ at $x \in N$ as a subspace of the tangent space $T_{f(x)}(M)$ of $M$. To avoid repetition, we will fix the ranges of the following indices, $1 \leq i, j, k \leq n ; n+1 \leq \alpha, \beta, \gamma \leq$ $n+p ; n+p+1 \leq s \leq n+p+q ; n+1 \leq \rho, \sigma \leq n+p+q ; 1 \leq A, B, C, D \leq n+p$. Ranges of other indices will vary and will be defined accordingly.

1. Exterior differential systems. In this section, we will give a résume of Cartan-Kähler theory of exterior differential systems, mainly to establish some notations and to state the existence theorems which will be used later. Details 
and proofs can be found in [3] and [7]. For an interesting modern survey of the theory, one can refer to [5].

An exterior differential system (or simply differential system) $I$ on a manifold $M$ is an ideal (finitely generated) in the ring of analytic differential forms on $M$ which is also closed under exterior differentiation, i.e. $d I C l$. We shall denote by $I_{\nu}$ the set of all forms of degree $\nu$ in $I$. Points of $M$, at which

$$
I_{0}=0
$$

constitute an analytic subvariety $\mathcal{E}^{0}(I)$ of $M$ whose points will be called integral points or 0-dim integral elements of $I$. Restricted to a point $m \in M, I$ defines a system of exterior equations $I(m)$ in the vector space $T_{m}(M)$. At an integral point $m$, a $k$-dim subspace $E$ of $T_{m}(M)$ is called a $k$-dim integral element of $I$ if $E$ annihilates $I(m)$. A submanifold $N$ of $M$ is called an integral manifold of $I$ if for every $m \in N, T_{m}(N)$ is an integral element of $l$, that is, the restriction (i.e. pull back) of $I$ to $M$ vanishes identically. For definiteness, let $M$ now be a manifold of dimension $n+p$ (later on, we will also consider differential systems on manifolds of other dimensions) and $\theta_{1}, \ldots, \theta_{n}$ be $n$ independent Pfaffian forms defined on M. An important problem in the application of Cartan-Kähler theory is to see if a differential system $I$ has an integral manifold on which

$$
\theta_{1} \wedge \ldots \wedge \theta_{n} \neq 0
$$

To study this problem we adjoin to $\theta_{1}, \ldots, \theta_{n}, p$ Pfaffian forms $\theta_{n+1}, \ldots, \theta_{n+p}$ such that $\theta_{1} \wedge \ldots \wedge \theta_{n+p} \neq 0$. Then the forms in $I$ can be expressed in terms of the $\theta$ 's. We put

$$
\theta_{a}=\sum_{i} b_{a_{i}} \theta_{i}
$$

and denote by $b_{i}$ the vector whose components are $b_{n+1, i}, \ldots, b_{n+p, i}$. Substitute (1.3) into the forms of $I_{j}$ and let $I_{j}\left(m, b_{1}, \ldots, b_{j}\right)$ denote the set of coefficients of $\theta_{1} \wedge \ldots \wedge \theta_{j}$ in these forms. Clearly every equation of the set

$$
I_{j}\left(m, b_{1}, \cdots, b_{j}\right)=0 \quad(j=1, \cdots, n)
$$

is linear in each of the variables $b_{\nu}(\nu=1, \ldots, j)$. At an integral point $m, a$ system of solutions $\left(m^{\circ}, b_{1}^{\circ}, \ldots, b_{n}^{\circ}\right)$ of $(1.1)$ and (1.4) defines an $n$-dim integral element of $I$ by (1.3) with $b_{a_{i}}=b_{a_{i}}^{\circ}$. We will call an integral point $m$ of $I$ simple if there exists a neighborhood $\mathcal{U}$ of $m$ in $M$ such that $\mathscr{G}^{0}(I) \cap \mathcal{U}$ is a submanifold of $\mathcal{U}$ of dimension $r_{0}$ with equations (1.1). We can now give sufficient conditions for such an integral manifold of $I$ to exist.

Definition 1.1. (a) $I$ is said to be involutive with independent variables $\left\{\theta_{1}, \ldots \theta_{n}\right\}$ (an ordered set) at a point $m^{\circ}$ if there exists a system of solutions 
$\left(m^{\circ}, b_{1}^{\circ}, \ldots, b_{n}^{\circ}\right)$ of (1.1) and (1.4) such that (i) $m^{\circ}$ is a simple integral point of $I ;$ (ii) in a neighborhood $W$ of $\left(m^{\circ}, b_{1}^{\circ}, \ldots b_{n}^{\circ}\right)$ in $M \times \mathbf{R}^{n p}$, the equation (1.4) reduces to $(n+p)-r_{j}-j$ independent linear equations with respect to $b_{\alpha_{j}}$ after taking account of the equations

$$
I_{j-1}\left(m, b_{1}, \cdots, b_{j-1}\right)=0 .
$$

(b) Let $E_{n}$ be the $n$-dim integral element defined by a system of solutions $\left(m^{\circ}, b_{1}^{\circ}, \ldots b_{n}^{\circ}\right)$ of (1.1) and (1.4) as in (a) and $E_{j}$ be the subspace of $E_{n}$ on which $\theta_{j+1}=\cdots=\theta_{n}=0$. Then we have a chain of integral elements of $I$

$$
\left\{m^{\circ}\right\}=E_{0} \subset E_{1} \subset \cdots \subset E_{n-1} \subset E_{n} .
$$

Any chain of integral elements of $I$ which can be obtained in this way by a suitable choice of the independent Pfaffian forms $\left\{\theta_{1}, \ldots, \theta_{n}\right\}$ in (a) is called an $n$-dim regular integral chain of $I, E_{\mu}(\mu=0,1, \ldots, n)$ is called its $\mu$ th component. $A(\nu-1)$-dim integral element $E_{\nu-1}$ of $I$ is called regular if it is the $(\nu-1)$ th component of an $n$ - $\operatorname{dim}(n \geq \nu)$ regular integral chain of $I$. In this case, the nonnegative integer $r_{\nu}=r_{\nu}\left(E_{\nu-1}, I\right)$ as defined in (a) is called the character of $E_{\nu-1}$.

Remark 1.1. It is clear that, in Definition 1.1 (a), $I$ is also involutive at any integral point sufficiently close to $m^{\circ}$ and also that if $E_{n}$ is another integral element of $I$ defined by $\left(m, b_{1}, \ldots, b_{n}\right)$ sufficiently close to $\left(m^{\circ}, b_{1}^{\circ}, \ldots, b_{n}^{\circ}\right)$, then the integral chain constructed on $E_{n}$ as in (b) is also regular.

Remark 1.2. The definitions of regular integral elements and regular integral chains given above are equivalent to other existing definitions of such, but they are more convenient for our present purpose.

Remark 1.3. For a regular $(\nu-1)$-dim integral element $E_{\nu-1}$ at a point $m$, the set of all vectors $Y \in T_{m}(M)$ such that $Y$ spans with $E_{\nu-1}$ an integral element of $I$ is called the polar space $H\left(E_{\nu-1}, I\right)$ of $E_{\nu-1}$; it is a subspace of $T_{m}(M)$ of dimension $r_{\nu}\left(E_{\nu-1}, I\right)+\nu$. In other words, the set of all $\nu$-dim integral elements which extend $E_{\nu-1}$ locally depends on $r_{\nu}\left(E_{\nu-1}, I\right)$ parameters.

Definition 1.2. A $\mu$-dim integral manifold $N$ of $I$ is called regular if, for every $m \in N, T_{m}(N)$ is a regular integral element of $I$ and it is called a general solution if, for every $m \in N, T_{m}(N)$ contains a $(\mu-1)$-dim regular integral element of $I$.

The fundamental theorem of Cartan-Kähler theory can be stated as follows:

Theorem 1.1. Let I be an exterior differential system on a manifold $M$ of dimension $n+p$. Let $N_{\nu-1}$ be a $(\nu-1)$-dim regular integral manifold of $I$ 
and $r_{\nu}$ the character of $T_{m^{\circ}}\left(N_{\nu-1}\right)$ at a point $m^{\circ} \in N_{\nu-1}$. Let $F$ be a $\left(n+p-r_{\nu}\right)$-dim submanifold of $M$ sucb that it contains $N_{\nu-1}$ and $T_{m^{\circ}}(F)$ contains a unique $\nu$-dim integral element $E_{\nu}$ of I which extends $E_{\nu-1}$, that is, $T_{m \circ}(F) \cap H\left(E_{\nu-1}, I\right)=E_{\nu}$. Then in a sufficiently small neigbborbood $\mathcal{U}$ of $m^{\circ}$, there exists a unique $\nu$-dim integral manifold $N_{\nu}$ of I sucb that $F \cap U \supset N_{\nu} \supset$ $\mathcal{U} \cap N_{\nu-1}$ and $T_{m}^{\circ}(N)=E_{\nu}$.

By applying Theorem 1.1 several times, we can prove the following theorem:

Theorem 1.2. Let I be a differential system on a manifold $M$ of dimension $n+p$ which is involutive with independent variables $\left\{\theta_{1}, \ldots, \theta_{n}\right\}$ at $m^{\circ}$. Let $E_{n}$ be the n-dim integral element defined by a system of solutions $\left(m^{\circ}, b_{1}^{\circ}, \ldots\right.$, $\left.b_{n}^{\circ}\right)$ of (1.1) and (1.4) which satisfies the conditions in Definition 1.1 (a).

Then in a sufficiently small neighborbood of $m^{\circ}$, there exist general solutions of $I$ on whicb $\theta_{1} \wedge \ldots \wedge \theta_{n} \neq 0$. In particular, there exists a general solution $N$ of I through $m^{\circ}$ such that $T_{m}^{\circ}(N)=E_{n}$.

Remark 1.4. The general solution $N$ in Theorem 1.2 is in general not uniquely determined. Classically this is described as depending on certain arbitrary choices of functions $[3$, p. 75]. For a modern and more precise description of such, see [9].

2. The Main Theorem. Let $I$ be an exterior differential system on a manifold $M$ of dimension $n+p$. Since all the results in this paper are local, we will assume for simplicity that $I_{0}=\varnothing$, i.e. the ideal $I$ contains no scalar function. In order to motivate the following discussions, we will sketch a proof of the following facts (see also [5, Theorem 3.1]).

For a one-parameter family of $n$-dim submanifolds of $M$ defined by $f: N \times$ $(-1,1) \rightarrow M$ (i.e. for every $t \in(-1,1), f(N \times t)$ is an $n$-dim submanifold of $M)$, the vector field $\mathbf{v}$ on $f(N \times 0)$ defined by

$$
\mathbf{v}(f(y, 0))=f_{*}(\partial / \partial t(y, 0))
$$

(where $\partial / \partial t$ is the standard vector field on the interval $(-1,1)$ ) is called the deformation vector field of the one-parameter family on $f(N \times 0)$.

Proposition 2.1. Suppose $f: N \times(-1,1) \rightarrow M$ defines a one-parameter family of integral manifolds of $I$. Then its deformation vector field $\mathbf{v}$ on $f(N \times 0)$ satisfies the following system of differential equations:

$$
\left.\left.0=\partial f^{*}(\theta) /\left.\partial t\right|_{t=0}=d f_{0}^{*}(v\rfloor \theta\right)+f_{0}^{*}(\mathrm{v}\rfloor d \theta\right) \text { for all } \theta \in I,
$$

where $\rfloor$ denotes the interior product of a tangent vector and a covector and $f_{0}: N \rightarrow M$ is the map defined by $f_{0}(y)=f(y, 0)$ for $y \in N$. 
Proof. We will prove that for any form $\theta$ defined on $M$, we have

$$
\left.\left.\partial f^{*}(\theta) /\left.\partial t\right|_{t=0}=d f_{0}^{*}(\mathrm{v}\rfloor \theta\right)+f_{0}^{*}(\mathrm{v}\rfloor d \theta\right) .
$$

It is obvious that (2.3) is true if $\theta$ is a zero-form. By taking exterior derivative on both sides of (2.3), we can see that if it is true for $\theta$ it is also true for $d \theta$. Using the facts that both the interior multiplication and exterior differentiation are antiderivations in the ring of differential forms on $M$, it follows by a straightforward computation that if (2.3) is true for forms $\theta_{1}$ and $\theta_{2}$ it is also true for the form $\theta_{1} \wedge \theta_{2}$. Now, since the ring of differential forms can be locally built up from 0 -forms using $d$ and $\Lambda$, the proof of (2.3) is completed. $f^{*}(\theta)$ is identically zero for all $\theta \in I$; therefore (2.2) also follows. Q. E. D.

Definition 2.1. Let $g: N \rightarrow M$ define an integral manifold of $I$. A vector field v: $g(N) \rightarrow T(M)$ defined on $g(N)$ is called an $I$-field if it satisfies the following system of differential equations, called the equations of variation of $I$ on $g(N)$ :

$$
\left.\left.d g^{*}(\mathrm{v}\lrcorner \theta\right)+g^{*}(\mathbf{v}\lrcorner d \theta\right)=0 \text { for all } \theta \in I .
$$

Remark 2.1. In the computation of the equations of variation it will be sufficient to consider the forms in any set of generators of $I$ as an ideal.

The main result of this paper can be stated as follows.

Main Theorem. Let $N$ be an n-dim general solution of an exterior differential system I on a manifold $M$ of dimension $n+p$. Let $\mathrm{v}: N \rightarrow T(M)$ be an I-field on $N$ sucb that for $m \in N, \mathrm{v}(m)$ is either the zero vector or it is not in $T_{m}(M)$. Then for every $m^{\circ} \in N$, there exists in a sufficiently small neighborbood $2^{m}$ of $m^{\circ}$ in $M$ a one-parameter family of $n$-dim integral manifolds ${ }^{t} N$ of $I$, where $t \in(-\epsilon, \epsilon)$ and $\epsilon>0$, sucb that ${ }^{0} N=N \cap \mathcal{Q}$ and the deformation vector field of ${ }^{t} N$ on ${ }^{0} N$ is equal to the restriction of $\mathrm{v}$ to ${ }^{0} N$.

3. Normal exterior differential systems. Under the assumptions of the Main Theorem it is always possible to choose a system of local coordinates $\left\{x_{i}, z_{\alpha}\right\}$ in a sufficiently small neighborhood $\mathcal{U}$ of $m^{\circ}$ in $M$ such that

(a) $N \cap \mathcal{U}$ can be defined nonparametrically as

$$
z_{a}=g_{a}\left(x_{1}, \cdots, x_{n}\right)
$$

and on $N \cap \mathcal{U}$ can be represented as

$$
\mathbf{v}(m)=\sum_{a} \tau_{a}\left(x_{1}(m), \cdots, x_{n}(m)\right)\left(\frac{\partial}{\partial z_{a}}\right)_{m},
$$

for some analytic functions $g_{\alpha}$ and $\tau_{\alpha}$ defined on a suitable open set in $\mathbf{R}^{n}$; 
(b) restricted to $\mathcal{U}, I$ is involutive with independent variables $\left\{d x_{1}, \ldots\right.$, $\left.d x_{n}\right\}$ at every point of $\mathcal{U}$.

Keeping the notations in $\S_{1}$ except with $\theta_{\alpha}$ and $\theta_{i}$ replaced by $d z_{\alpha}$ and $d x_{i}$ (respectively), if we put

$$
b_{\alpha_{i}}^{\circ}=\left(\partial g_{\alpha} / \partial x_{i}\right)\left(x_{1}\left(m^{\circ}\right), \ldots, x_{n}\left(m^{\circ}\right)\right),
$$

then $\left(m^{\circ}, b_{1}^{\circ}, \ldots, b_{n}^{\circ}\right)$ which defines the integral element $T_{m^{\circ}}(N)$ of $I$ satisfies the conditions of Definition 1.1 (a). Let $\mathcal{P}(I)$ be the exterior differential system defined on the neighborhood $W$ of $\left(m^{\circ}, b_{1}^{\circ}, \ldots, b_{n}^{\circ}\right)$ which is generated by the left-hand side of the following equations:

$$
\begin{aligned}
I_{j}\left(m, b_{1}, \ldots, b_{j}\right) & =0, \\
d I_{j}\left(m, b_{1}, \ldots, b_{j}\right) & =0, \\
d z_{a}-\sum_{i} b_{a_{i}} d x_{i} & =0, \\
\sum_{i} d b_{a i} \wedge d x_{i} & =0 .
\end{aligned}
$$

One can easily recognize that $\mathcal{P}(I)$ is the total prolongation of $I$ (restricted to $\mathcal{U})$ as defined in [11]. It follows from Theorem 2 in [11] that $\mathcal{P}(I)$ is also involutive with independent variables $\left\{d x_{1}, \ldots, d x_{n}\right\}$ at every point of $W$. By a straightforward computation, we can see that, upon setting

$$
\begin{aligned}
b_{a_{i}} & =\partial z_{a} / \partial x_{i}, \\
\tau_{a_{i}} & =\partial \tau_{\alpha} / \partial x_{i},
\end{aligned}
$$

(3.1) and (3.8) define a general solution of $\mathcal{P}(I)$ and

$$
\sum_{a} \tau_{a} \frac{\partial}{\partial z_{a}}+\sum_{a, i} \tau_{\alpha i} \frac{\partial}{\partial b_{\alpha i}}
$$

defines a $\mathcal{P}(I)$-field on it. Let $\pi: W \rightarrow M$ be the map defined by

$$
\pi\left(m, b_{1}, \ldots, b_{n}\right)=m \text {. }
$$

Similarly we can also verify that, if $w$ is a $\mathscr{P}(I)$-field on an integral manifold $B$ of $\mathcal{P}(I)$ on which $d x_{1} \wedge \ldots \wedge d x_{n} \neq 0$, then $\pi_{*}(w)$ is an $I$-field on the integral manifold $\pi(B)$ of $I$. It is also well known that (3.4) defines a submanifold $\mathcal{E}^{n}(I)$ of $W$. In fact, we can select $q=\sum_{j=1}^{n}\left(r_{j}+j-n\right)$ of the $b^{\prime}$ 's such that they form together with $\left\{x_{i}, z_{\alpha}\right\}$ a system of coordinates of $\mathcal{E}^{n}(I)$ [7, p. 42]. The restriction of $\mathscr{P}(I)$ to $\mathscr{E}^{n}(I)$ is also involutive with independent variables $\left\{d x_{1}, \ldots, d x_{n}\right\}$. Note also that a $\mathcal{P}(I)$-field must be tangent to $\mathcal{E}^{n}(I)$. The above considerations lead us to consider a class of Pfaffian systems which will be called normal systems. 
Definition 3.1. An exterior differential system $I$ on an open set $l\left(C \mathbf{R}^{n+p+q}\right.$ is called normal with $n$ independent variables, $p$ primary dependent variables and $q$ secondary dependent variables, or simply a normal system of type $(n, p, q)$ if it satisfies the following conditions:

(i) $I_{0}=\emptyset$.

(ii) There exists a system of coordinates $\left\{x_{i}, z_{\alpha}, z_{s}\right\}$ of 6 , called normal coordinates of $I$, and functions $B_{a_{i}}$ on $W$ so that $I$ is generated as an ideal by the following differential forms

$$
d z_{a}+\sum_{i} B_{a_{i}} d x_{i}, \quad \sum_{i} d B_{a_{i}} \wedge d x_{i}
$$

and $I$ is also involutive with independent variables $\left\{d x_{1}, \ldots, d x_{n}\right\}$ at every point of $10 .\left\{z_{\alpha}\right\}$ and $\left\{z_{s}\right\}$ are called respectively primary and secondary dependent variables.

Definition 3.2. A normal solution of a normal system $I$ of type $(n, p, q)$ with a fixed system of normal coordinates $\left\{x_{i}, z_{\alpha}, z_{s}\right\}$ is an $n$-dim integral manifold $N$ of $I$ on which $d x_{1} \wedge \ldots \wedge d x_{n} \neq 0$ and such that for $m \in N$, the chain of integral elements on $T_{m}(N)$ obtained by setting successively $d x_{k}=d x_{k+1}=\cdots=$ $d x_{n}=0$ is an $n$-dim regular integral chain $I$.

We can see now that to prove the Main Theorem it is sufficient to prove it for normal solution $N$ of a normal system $I$ of type $(n, p, q)$ with an $I$-field $\mathbf{v}$ on it such that

(i) with respect to a system of normal coordinates $\left\{x_{i}, z_{\alpha}, z_{s}\right\}$ of $I$ for which $N$ is a normal solution, $N$ can be represented nonparametrically in an open subset of 6 as

$$
z_{\tau}=g_{\sigma}\left(x_{1}, \ldots, x_{n}\right),
$$

(ii) $\mathbf{v}$ can be represented as

$$
\mathbf{v}(m)=\sum_{\sigma} \tau_{\sigma}\left(x_{1}(m), \ldots, x_{n}(m)\right)\left(\frac{\partial}{\partial z_{\sigma}}\right)_{m}
$$

for some analytic functions $g_{\sigma}$ and $\tau_{\sigma}$ defined on a suitable open set of $\mathbf{R}^{n}$.

Definition 3.3. A normal solution $N$ of a normal system $I$ of type $(n, p, q)$ together with an I-field $\mathbf{v}$ on it which have properties as in (i) and (ii) above is called a set of normal data of $I$.

4. Two associated differential systems. In this section $I$ denotes a normal system of type $(n, p, q)$ on an open set $W$ in $\mathbf{R}^{n+p+q}$ with a fixed choice of normal coordinates $\left\{x_{i}, z_{\alpha}, z_{s}\right\}$ and it is generated as an ideal by (3.12). Let $\xi_{\alpha}$ be the coordinates of $\mathbf{R}^{p}$ and $t$ the coordinate of the open interval $(-1,1)$. Denote by $\tilde{I}$, the differential system on $60 \times \mathbf{R}^{p} \times(-1,1)$ generated by the following differential forms, 


$$
d z_{a}+\sum_{i} B_{a_{i}} d x_{i}-\xi_{\alpha} d t, \quad \sum_{i} d B_{\alpha_{i}} \wedge d x_{i}-d \xi_{\alpha} \wedge d t
$$

Let

$$
\pi_{1}: 10 \times \mathbf{R}^{p} \times(-1,1) \rightarrow(10
$$

be the natural projection map. We will denote by $\widetilde{m}\left(=\left(m, \xi_{\alpha}, t\right)\right)$ a point of $\mathbb{C}_{x}$ $\mathbf{R}^{p} \times(-1,1)$ and that of 60 by $m$.

Proposition 4.1. Let

$$
\pi_{1}\left(m^{\circ}\right)=E_{0} \subset E_{1} \subset \cdots \subset E_{n-1} \subset E_{n}
$$

be an n-dim regular integral chain of I such that $d x_{1} \wedge \cdots \wedge d x_{j} \neq 0$ on $E_{j}$. Then there exists a chain of integral elements of $\tilde{I}$,

$$
\tilde{m}^{\circ}=\widetilde{E}_{0} \subset \widetilde{E}_{1} \subset \cdots \subset \widetilde{E}_{n} \subset \widetilde{E}_{n+1},
$$

such that $\pi_{1 *}\left(\widetilde{E}_{j+1}\right)=E_{j}$, dt $\neq 0$ on $\widetilde{E}_{1}$ and $d t \wedge d x_{1} \wedge \ldots \wedge d x_{j} \neq 0$ on $\widetilde{E}_{j+1}$. Furthermore any sucb integral chain is a regular $(n+1)$-dim integral chain of $I$ and

$$
r_{1}\left(\tilde{E}_{0}, I\right)=n+p+q, \quad r_{j+1}\left(\tilde{E}_{j}, I\right)=r_{j}\left(E_{j-1}, I\right) .
$$

It follows that $\tilde{I}$ is involutive with independent variables $d t, d x_{1}, \ldots, d x_{n}$ at any point of $60 \mathbf{R}^{p} \times(-1,1)$.

Proof. Any point $\tilde{m} \in \mathcal{W} \times \mathbf{R}^{p} \times(-1,1)$ is a simple integral point of $\tilde{I}$. We put

$$
d z_{\sigma}=b_{\sigma 0} d t+\sum_{i} b_{\sigma i} d x_{i}, \quad d \xi_{\alpha}=a_{a 0} d t+\sum_{i} a_{a i} d x_{i} .
$$

Denote by $b_{\nu}(\nu=0,1, \ldots, n)$ the vector whose components are $b_{\sigma \nu}, a_{\alpha \nu}$. Substituting (4.6) into $\tilde{I}$, we can see, by a straightforward computation, that $\tilde{I}_{1}\left(m, b_{0}\right)=0$ is generated by the following equations

$$
b_{a 0}-\xi_{a}=0
$$

and in general, $\tilde{I}_{j+1}\left(m, b_{0}, b_{1}, \ldots, b_{j}\right)=0$ after taking account of $\tilde{I}_{j}\left(m, b_{0}, b_{1}, \ldots, b_{j-1}\right)=0$ is generated by the following equations ( $j$ is fixed),

$$
b_{a_{j}}+B_{a_{j}}=0
$$

$$
\sum_{\sigma}\left(B_{\alpha \mu ; \sigma^{b}} \sigma_{\sigma_{j}}-B_{\left.\alpha_{j ;} \sigma^{b}{ }_{\sigma \mu}\right)+B_{\alpha \mu ; j}-B_{a j ; \mu}=0 \quad(\mu=1, \ldots, j-1)}\right.
$$

$$
\sum_{\sigma} B_{a j ; \sigma^{b 0}}+a_{a j}=0
$$


where we have set $B_{a_{j} ; \sigma}=\partial B_{\alpha_{j}} / \partial z_{\sigma}$ and $B_{a_{j} ; i}=\partial B_{\alpha_{j}} / \partial x_{i}$. If we set $d z_{\sigma}=$ $\Sigma_{i} b_{\sigma i} d x_{i}$ in $l$ and let $b_{i}$ be the vector whose components are $b_{\sigma i}$, then it follows by a similar computation as above that $I_{j}\left(\pi_{1}(m), b_{1}, \ldots, b_{j}\right)=0$ is also generated by (4.8) after taking account of $I_{j-1}\left(\pi_{1}(m), b_{1}, \cdots b_{j-1}\right)=0$. Therefore, if $(4.3)$ is defined by $\left(\pi_{1}\left(m^{\circ}\right), b_{1}^{\circ}, \ldots, b_{n}^{\circ}\right)$, we can extend it to an $(n+1)$ dim regular integral chain (4.4) of $\tilde{I}$ by choosing $b_{s 0}, a_{a 0}$ arbitrarily, and define $b_{\alpha 0}, a_{\alpha_{j}}$ by (4.7) and (4.9) respectively. It follows from our constructions that $\pi_{1 *}(\widetilde{E})=\pi_{1}(\tilde{m})$ and $\pi_{1 *}\left(\widetilde{E}_{j+1}\right)=\widetilde{E}_{j}$. (4.5) now follows readily from the definition of the character of a regular integral element and the fact that (4.7) as a linear system in $b_{\alpha 0}$ as well as (4.9) as a linear system in $a_{a_{j}}$ are of rank $p$. Q. E. D.

Remark 4.1. An examination of the above proof shows that for any $(\mu+1)$ $\operatorname{dim}$ integral element $\widetilde{E}_{\mu+1}$ of $\widetilde{I}$ which extends $E_{\mu}$, i.e. $\pi_{1 *}\left(E_{\mu+1}\right) \approx E_{\mu}$, there $\underset{\sim}{\text { exists a unique }}(\mu+2)$-dim integral element $\widetilde{E}_{\mu+2}$ of $\tilde{I}$ such that $\widetilde{E}_{\mu+2}$ ) $\widetilde{E}_{\mu+1}$ and $\pi_{1 *}\left(\widetilde{E}_{\mu+2}\right)=E_{\mu+1}, \mu=1, \ldots, n-1$.

Remark 4.2. If $\tilde{N}$ is an $n$-dim integral manifold of $\tilde{I}$ on which $d t \wedge d x_{1} \wedge$ $\ldots \wedge d x_{n} \neq 0$, then $\pi_{1}(\tilde{N})$ is a one-parameter family of integral manifolds of $I$ on which $d x_{1} \wedge \ldots \wedge d x_{n} \neq 0$.

$\tilde{I}$ will be used to construct the one-parameter family of integral manifolds we need. But to prove the deformation vector field of the one-parameter family to be constructed is the given I-field, we need to consider another associated differential system.

Let $\xi_{\sigma}$ be coordinates of $\mathbf{R}^{p+q}$. Denote by $m^{\prime}\left(=\left(m, \xi_{\sigma}\right)\right)$ a point of $6 \times$ $\mathbf{R}^{p+q}$ and also by $\pi_{2}: W \times \mathbf{R}^{p+q} \rightarrow W$ the natural projection map. Define a vector field $\xi$ on $10 \times \mathbf{R}^{p+q}$ by

$$
\xi\left(m^{\prime}\right)=\sum_{\sigma} \xi_{\sigma}\left(\frac{\partial}{\partial z_{\sigma}}\right)_{m} .
$$

Then let $I^{\prime}$ be the exterior differential system on $W \times \mathrm{R}^{p+q}$ which is generated as an ideal by the following differential forms,

$$
\begin{gathered}
d z_{a}+\sum_{i} B_{a_{i}} d x_{i}, \quad \sum_{i} d B_{a_{i}} \wedge d x_{i^{\prime}} \\
\left.\left.d\{\xi\lrcorner\left(d z_{\alpha}+\sum_{i} B_{a_{i}} d x_{i}\right)\right\}+\{\xi\lrcorner \sum_{i} d B_{a_{i}} \wedge d x_{i}\right\}, \\
\left.d\{\xi\lrcorner \sum_{i} d B_{a i} \wedge d x_{i}\right\} .
\end{gathered}
$$


Re mark 4.3. With functions $g_{\sigma}$ and $\tau_{\sigma}$ as in (3.13) and (3.14), the submanifold $N^{\prime}$ of $6 \times \mathbf{R}^{p+q}$ defined by

$$
\begin{aligned}
& z_{\sigma}=g_{\sigma}\left(x_{1}, \ldots, x_{n}\right), \\
& \xi_{\sigma}=\tau_{\sigma}\left(x_{1}, \ldots, x_{n}\right)
\end{aligned}
$$

in an $n$-dim integral manifold of $I^{\prime}$. This follows readily from the fact that if we substitute (4.13) into (4.12), we have the equations of variation on the integral manifold $N$ of $I$ defined by (4.13) for the special type of vector field which can be represented as the right-hand side of (4.10). Conversely, for any $n$-dim integral manifold $N^{\prime}$ of $I^{\prime}$ defined (4.13) and (4.14) for some analytic functions $g_{\sigma}$ and $\tau_{\sigma}, \Sigma_{\sigma} \tau_{\sigma} \partial / \partial z_{\sigma}$ is an $l$-field on the integral manifold $\pi_{2}\left(N^{\prime}\right)$ of $I$.

We will prove later on that at any point $m^{\prime}, I^{\prime}$ is also involutive with independent variables $\left\{d x_{1}, \ldots, d x_{n}\right\}$. However, we will conclude this section by making some important observations related to $I^{\prime}$. We put

$$
\begin{aligned}
& d z_{\sigma}=\sum_{i} b_{\sigma_{i}} d x_{i}, \\
& d \xi_{\sigma}=\sum_{i} a_{\sigma_{i}} d x_{i} .
\end{aligned}
$$

Denote by $b_{i}^{\prime}$ the vector whose components are $b_{\sigma_{i}}, a_{\sigma_{i}}$ and as before, $b_{i}$ the vector whose components are $b_{\sigma i}$. By a straightforward computation, we can see that $I_{k}^{\prime}\left(m^{\prime}, b_{1}^{\prime}, \ldots, b_{k}^{\prime}\right)=0$ after taking into account that $I_{k-1}^{\prime}\left(m^{\prime}, b_{1}^{\prime}, \ldots, b_{k-1}^{\prime}\right)$ $=0$ is generated by the equations ( $k$ being fixed):

$$
b_{a k}+B_{a k}=0 \text {, }
$$

$\left(4.17_{k}\right) \sum_{\sigma}\left(B_{a \mu ; \sigma^{b} b}-B_{a k ; \sigma^{b} \sigma_{\mu}}\right)+B_{a \mu ; k}-B_{a k ; \mu}=0 \quad(\mu=1, \ldots, k-1)$

$$
\begin{aligned}
& a_{a k}+\sum_{\sigma} B_{a k ; \sigma} \xi_{\sigma}=0 \\
& \sum_{\sigma}\left(B_{a \mu ; \sigma} a_{\sigma k}\right. \\
& \left.-B_{a k ; \sigma} a_{\sigma \mu}\right)+\sum_{\sigma, \rho}\left(B_{a \mu ; \sigma ; \rho} \rho_{\rho k}-B_{a k ; \sigma ; \rho} b_{\rho \mu}\right) \xi_{\sigma} \\
& \quad+\sum_{\sigma}\left(B_{a \mu ; \sigma ; k}-B_{a k ; \sigma ; \mu}\right) \xi_{\sigma}=0 \quad(\mu=1, \ldots, k-1)
\end{aligned}
$$

where we have set $B_{\alpha \mu ; \sigma ; \rho}=\partial^{2} B_{\alpha \mu} / \partial z_{\sigma} \partial z_{\rho}, B_{\alpha \mu ; \sigma ; i}=\partial^{2} B_{\alpha \mu} / \partial z_{\sigma} \partial x_{i}$.

Remark 4.4. It is easy to see that $I_{k}\left(\pi_{2}\left(m^{\prime}\right), b_{1}, \ldots, b_{k}\right)=0$ is generated by $\left(4.17_{k}\right)$ after taking account of $I_{k-1}\left(\pi_{2}\left(m^{\prime}\right), b_{1}, \ldots, b_{k-1}\right)=0$. If we consider $\left(4.17_{k}\right)$ and $\left(4.18_{k}\right)$ as linear equations in $b_{\sigma k}$ and $a_{\sigma k}$ respectively, then the coefficient matrix of $b_{\sigma k}$ in $\left(4.17_{k}\right)$ is exactly the same as the coefficient matrix of $a_{\sigma k}$ in $\left(4.18_{k}\right)$. 
5. Proof of the Main Theorem. In this section, $I$ will denote the same normal system of type $(n, p, q)$ as in the previous one. We will now prove the Main Theorem for a normal solution (Definition 3.2) $N$ of $I$ with an $I$-field $\mathbf{v}$ on it such that they can be defined by a set of normal data (Definition 3.3) of $I$. As noted earlier, this will prove the Main Theorem in general.

Let $\left\{x_{i}, z_{\alpha}, z_{s}\right\}$ be the fixed normal coordinates of $I$. At any point $m \in \mathcal{W}$, let $W_{\nu}(m)(\nu=1, \ldots, n-1)$ be the submanifold of 6 defined by

$$
x_{\nu+1}=x_{\nu+1}(m), \cdots, x_{n}=x_{n}(m) .
$$

We will simply write $6_{\nu}$ when the point $m$ is clear in the context. Denote by $\Re_{\nu} I(m)$ the differential system on $W_{\nu}(m)$ obtained by restricting $I$ to $\mathcal{O}_{\nu}(m)$. One can easily verify that $\mathbb{R}_{\nu} I(m)$ is a normal system of type $(\nu, p, q)$ on $\mathbb{W}_{\nu}$. In fact, $\left\{x_{1}, \ldots, x_{\nu}, z_{n+1}, \ldots, z_{n+p}, z_{n+p+1}, \ldots, z_{n+p+q}\right\}$ is a system of normal coordinates of $\Re_{\nu} I(m)$ such that $\mathscr{R}_{\nu} I(m)$ is involutive with independent variables $\left\{d x_{1}, \ldots, d x_{\nu}\right\}$ at every point of $C_{\nu} ; z_{\alpha}$ and $z_{s}$ are still the primary and secondary independent variables respectively; the restriction of (3.12) to $W_{\nu}(m)$ also generates $R_{\nu} I(m)$ as an ideal. This is the only set of normal coordinates of $\mathcal{R}_{\nu} I(m)$ we will use. Let $R_{\nu} I^{\prime}(m)$ and. $R_{\nu} \tilde{I}(m)$ be the restrictions of $I^{\prime}$ and $\tilde{I}$ to $\mathscr{C}_{\nu}(m) \times \mathbf{R}^{p+q}$ and $\mathscr{C}_{\nu}(m) \times \mathbf{R}^{p} \times(-1,1)$ respectively. They are actually also the differential systems associated with $R_{\nu} I(m)$ (with respect to the normal coordinates of $\Re_{\nu} I(m)$ fixed above) as defined in the previous section. Therefore, by Proposition 4.1, $\Re_{\nu} \tilde{I}(m)$ is also involutive at every point of $60_{\nu} \times \mathbf{R}^{p} \times(-1,1)$. For uniformity, we will also denote by $\mathbb{W}_{0}(m)$ the submanifold of 6 defined by

$$
x_{1}=x_{1}(m), \cdots, x_{n}=x_{n}(m) .
$$

We will call any point of $W_{0}(\mathrm{~m})$ a normal solution or 0 -dim integral manifold of $R_{0} I(m)$ and any tangent vector at a point $m^{\circ} \in \mathcal{O}_{0}(m)$ of the form $\Sigma_{\sigma} \xi_{\sigma}^{\circ}\left(\partial / \partial z_{\sigma}\right)_{m^{\circ}}$ an $R_{0} I(m)$-field. A set of normal data of $R_{0} I(m)$ consists of a point of $\mathbb{Q}_{0}(\mathrm{~m})$ and an $\mathcal{R}_{0} I(m)$-field at that point. We also put $\mathcal{R}_{n} I(m)=I, \mathcal{R}_{n} I^{\prime}(m)=I^{\prime}, \mathbb{R}_{n} \tilde{I}(m)$ $=\tilde{I}$ and $W_{n}=6 \%$.

It follows directly from our definitions that a normal solution or a set of normal data of $R_{k} I(m)$ restricts to a normal solution or a set of normal data of $R_{k-1} I(m)$ respectively.

The Main Theorem will follow from this proposition:

Proposition 5.1. (1) Let $m^{\prime \circ} \in \mathbb{W} \times \mathbf{R}^{p+q}$ and $m^{\circ} \in \mathbb{W}$ be such that $\pi_{2}\left(m^{\prime \circ}\right)$ $=m^{\circ}$, i.e. $m^{\circ \circ}=\left(m^{\circ}, \xi_{\sigma}^{\circ}\right)$. Then, for $j=1, \ldots, n$, we bave $\mathbf{P}_{j}^{1}$ : If

$$
\left\{m^{\circ}\right\}=E_{0} \subset E_{1} \subset \cdots \subset E_{j-1} \subset E_{j}
$$


is a j-dim regular integral chain of $\mathscr{R}_{j} I\left(m^{\circ}\right)$ such that $d x_{1} \wedge \ldots \wedge d x_{\mu} \neq 0$ on $E_{\mu}(1 \leq \mu \leq j)$, then there exists a j-dim integral chain of $\mathscr{R}_{j} I^{\prime}\left(m^{\circ}\right)$,

$$
\left\{m^{\prime} \circ\right\}=E_{0}^{\prime} \subset E_{1}^{\prime} \subset \cdots \subset E_{j-1}^{\prime} \subset E_{j}^{\prime},
$$

which projects onto (5.3), that is, $\pi_{2 *}\left(E_{\mu}^{\prime}\right)=E_{\mu}$. Furthermore, any such integral chain of $\mathcal{R}_{j} I^{\prime}\left(m^{\circ}\right)$ is regular and

$$
r_{j}\left(E_{j-1}^{\prime}, \Re_{j} I^{\prime}\left(m^{\circ}\right)\right)=2 r_{j}\left(E_{j-1}, \Re_{j} I\left(m^{\circ}\right)\right) .
$$

(2) At any point $m^{\circ} \in$ W , we have, for $\nu=0,1, \ldots, n, \mathrm{P}_{\nu}^{2}$ : Let $N_{\nu}$ be a normal solution of $R_{\nu} I\left(m^{\circ}\right)$ which contains $m^{\circ}$ and $\mathrm{v}$ be an $R_{\nu} I\left(m^{\circ}\right)$-field on $N_{\nu}$ such that they can be defined by a set of normal data of $R_{\nu} I\left(m^{\circ}\right)$. Then in a sufficiently small neighborbood $\mathcal{U}_{\nu}$ of $m^{\circ}$ in $\mathcal{W}_{\nu}\left(m^{\circ}\right)$, there exists a one-parameter family of integral manifolds ${ }^{t} N_{\nu}$ of $R_{\nu} I\left(m^{\circ}\right)$, where $t \epsilon\left(-\epsilon_{\nu}, \epsilon_{\nu}\right)$ and $\epsilon_{\nu}>0$, sucb that ${ }^{0} N_{\nu}=N_{\nu} \cap \dot{U}_{\nu}$ and the deformation vector field of ${ }^{t} N_{\nu}$ on ${ }^{0} N_{\nu}$ coincides with the restriction of $\mathbf{v}$ to ${ }^{0} N_{\nu}$.

We will prove the proposition in the following order:

(a) $\mathbf{P}_{1}^{1}$ and $\mathbf{P}_{0}^{2}$ are both true,

(b) $\mathbf{P}_{j}^{1}$ and $\mathbf{P}_{j-1}^{2}$ imply $\mathbf{P}_{j}^{2}$,

(c) $\mathbf{P}_{j}^{1}$ and $\mathbf{P}_{j}^{2}$ imply $\mathbf{P}_{j+1}^{1}$.

We put for $j=1, \ldots, n$,

$$
d z_{\sigma}=\sum_{\mu=1}^{j} b_{\sigma \mu} d x_{\mu}, \quad d \xi_{\sigma}=\sum_{\mu=1}^{j} a_{\sigma \mu} d x_{\mu}
$$

in $R_{j} I(m)$ and $\mathcal{R}_{j} I^{\prime}(m)$ ( $b_{\mu}$ and $b_{\mu}^{\prime}$ will have the same meanings as in the previous section); we will find, for $\mu=1, \ldots, j,\left(R_{j} I(m)\right)_{\mu}\left(m, b_{1}, \ldots, b_{\mu}\right)$ and $\left(\Re_{j} I^{\prime}(m)\right)_{\mu}\left(m, b_{1}^{\prime}, \ldots, b_{\mu}^{\prime}\right)$ are the same as $I_{\mu}\left(m, b_{1}, \ldots, b_{\mu}\right)$ and $I_{\mu}^{\prime}\left(m, b_{1}^{\prime}, \ldots, b_{\mu}^{\prime}\right)$ respectively. For these reasons we will write $I_{\mu}(\ldots)$ and $I_{\mu}^{\prime}(\ldots)$ instead of $\left(\mathscr{R}_{j} I(m)\right)_{\mu}(\ldots)$ and $\left(\mathscr{R}_{j} I^{\prime}(m)\right)_{\mu}(\ldots)$ respectively.

Proof of $(\mathrm{a})$. Let $\left(4.17_{1}\right) *$ and $\left(4.18_{1}\right) *$ be respectively the linear equations in $b_{1}^{\prime}$ obtained by setting $m=m^{\circ}$ and $\xi_{\sigma}=\xi_{\sigma}^{\circ}$ in $\left(4.17_{1}\right)$ and $\left(4.18_{1}\right)$. Then the equations $I_{1}^{\prime}\left(m^{\prime}, b_{1}^{\prime}\right)=0$ are generated by $\left(4.17_{1}\right) *$ and $\left(4.18_{1}\right)^{*}$. By Remark 4.4 the equations $I_{1}\left(m^{\circ}, b_{1}\right)=0$ are also generated by $\left(4.17_{1}\right)^{*}$. Clearly $\left(4.17_{1}\right)^{*}$ and $\left(4.18_{1}\right) *$ as linear equations in $b_{\sigma 1}$ and $a_{\sigma 1}$ (resp.) are both compatible and of rank $p$. They are compatible and the ir ranks are independent of the points $m^{\prime}$ or $m$. Therefore $m^{\prime \circ}$ is a regular integral point of $\mathbb{R}_{1} I^{\prime}\left(m^{\circ}\right)$ and

$$
r_{1}\left(m^{\circ \circ}, R_{1} I^{\prime}\left(m^{\circ}\right)\right)=2 r_{1}\left(m^{\circ}, \mathcal{R}_{1} I\left(m^{\circ}\right)\right)=2 q \text {. }
$$

This proves $\mathbf{P}_{1}^{1}$. As for $\mathbf{P}_{0}^{2}$, let $\mathbf{v}=\Sigma_{\sigma} \xi_{\sigma}^{\circ}\left(\partial / \partial z_{\sigma}\right)_{m^{\circ}}$. The ${ }^{t} N_{0}$ can be defined by any analytic mapping $\alpha:\left(-\epsilon_{0}, \epsilon_{0}\right) \rightarrow \omega_{0}$ such that $\alpha(0)=m^{\circ}, \alpha^{\prime}(0)=$ $\Sigma_{\sigma} \xi_{\sigma}^{\circ}\left(\partial / \partial z_{\sigma}\right)_{m} \circ$ and $\epsilon_{0}>0$. 
In the remainder of this section $j$ will be fixed.

Proof of (b). We can assume that $x_{1}\left(m^{\circ}\right)=\cdots=x_{j}\left(m^{\circ}\right)=0$. In a neighborhood of $m^{\circ}$ in $6{ }_{j}\left(m^{\circ}\right), N_{j}$ and $\mathbf{v}$ are defined by

$$
\begin{aligned}
z_{\sigma} & =g_{\sigma}\left(x_{1}, \cdots, x_{j}\right), \\
\mathrm{v}(m) & =\sum_{\sigma} \tau_{\sigma}\left(x_{1}(m), \cdots, x_{j}(m)\right)\left(\frac{\partial}{\partial z_{\sigma}}\right)_{m} \text { for } m \in N_{j} .
\end{aligned}
$$

We put

$$
\xi_{\sigma}^{0}=\tau_{\sigma}(0, \ldots, 0)
$$

and denote by $m^{\circ} \circ$ the point $\left(m^{\circ}, \xi_{\sigma}^{\circ}\right) \in \mathbb{W}_{j} \times \mathbf{R}^{p+q}$. Then when we set

$$
\xi_{\sigma}=\tau_{\sigma}\left(x_{1}, \cdots, x_{j}\right) \text {, }
$$

(5.8) and (5.11) define in a neighborhood of $m^{\prime \circ}$ in $6{ }_{j} \times \mathbf{R}^{p+q}$ a $j$-dim integral manifold $N_{j}^{\prime}$ of $R_{j} I^{\prime}\left(m^{\circ}\right)$.

The restriction of $N_{j}$ to $W_{j-1}\left(m^{\circ}\right)$ defines a normal solution $N_{j-1}$ of $R_{j-1} I\left(m^{\circ}\right)$ and the restrictions of $(5.8)$ and $(5.9)$ to $W_{j-1}\left(m^{\circ}\right)$ define a set of normal data of $\mathcal{R}_{j-1} I\left(m^{\circ}\right)$. Therefore by $\mathbf{P}_{j-1}^{2}$, there exists in a neighborhood of $m^{\circ}$ in $6{ }_{j-1}\left(m^{\circ}\right)$ a one-parameter family of integral manifolds ${ }^{t} N_{j-1}$ of $\Re_{j-1} I\left(m^{\circ}\right)\left(t \in\left(-\epsilon_{j-1}, \epsilon_{j-1}\right), \epsilon_{j-1}>0\right)$ which can be defined as

$$
z_{\sigma}=f_{\sigma}\left(x_{1}, \ldots, x_{j-1} ; t\right)
$$

such that

$$
\begin{aligned}
f_{\sigma}\left(x_{1}, \ldots, x_{j-1} ; 0\right) & =g_{\sigma}\left(x_{1}, \ldots, x_{j-1}, 0\right), \\
\left(\partial f_{\sigma} / \partial t\right)\left(x_{1}, \ldots, x_{j-1} ; 0\right) & =\tau_{\sigma}\left(x_{1}, \ldots, x_{j-1}, 0\right)
\end{aligned}
$$

in a suitable neighborhood of the origin of $\mathbf{R}^{j-1}$. For every fixed $t$ $\Sigma_{\sigma}\left(\partial f_{\sigma} / \partial t\right)\left(\partial / \partial z_{\sigma}\right)$ is an $R_{j-1} I\left(m^{\circ}\right)$-field on ${ }^{t} N_{j-1}$. It follows by an easy computation that if we put

$$
\xi_{a}=\left(\partial f_{a} / \partial t\right)\left(x_{1}, \cdots, x_{j-1} ; t\right)
$$

then (5.12) and (5.14) define a j-dim integral manifold $\tilde{N}_{j-1}$ of $\mathbb{R}_{j-1} \tilde{I}\left(\mathrm{~m}^{\circ}\right)$ in a neighborhood of the point $\tilde{m}^{\circ}=\left(m^{\circ}, \xi_{\alpha}^{\circ}, 0\right) \in \widetilde{\sim}_{j-1} \times \sim^{p} \times(-1,1) . \widetilde{N}_{j-1}$ is also an integral manifold of $\mathcal{R}_{j} \tilde{I}\left(m^{\circ}\right)$. Put $\widetilde{E}_{j}=T_{\widetilde{m}^{\circ}}\left(\tilde{N}_{j-1}\right)$. Then it is clear that

$$
\pi_{1}\left(\tilde{m}^{\circ}\right)=m^{\circ}, \quad \pi_{1 *}\left(\tilde{E}_{j}\right)=T_{m} \circ\left(N_{j-1}\right) \text {. }
$$

Since $T_{m} \mathrm{o}\left(N_{j-1}\right)$ is a regular integral element of $\Re_{j} I\left(m^{\circ}\right)$, it follows readily from Proposition 4.1 and Remark 4.1 that $\widetilde{E}_{j}$ is also a regular integral element of $R_{j} \tilde{I}\left(m^{\circ}\right)$. If we put 


$$
r_{j}^{\circ}=r_{j}\left(T_{m} \circ\left(N_{j-1}\right), \Re_{j} I\left(m^{\circ}\right)\right)
$$

then the character of $\tilde{E}_{j}$ is given by

$$
r_{j+1}\left(\tilde{E}_{j}, \Re_{j} \tilde{I}^{\circ}\left(m^{\circ}\right)\right)=r_{j}^{\circ}
$$

If we set

$$
b_{\sigma \mu}^{\circ}=\left(\partial g_{\sigma} / \partial x_{\mu}\right)(0, \cdots, 0) \quad(\mu=1, \cdots, j)
$$

and denote by $b_{\mu}^{\circ}$ the vector whose components are $b_{\sigma \mu}^{\circ}$, then $\Gamma_{m} \mathrm{o}\left(N_{j-1}\right)$ and $T_{m}{ }^{\circ}\left(N_{j}\right)$ as integral elements of $\mathcal{R}_{j-1} I\left(m^{\circ}\right)$ and $\Re_{j} I\left(m^{\circ}\right)$ (resp.) are defined by $\left(m^{\circ}, b_{1}^{\circ}, \ldots, b_{j-1}^{\circ}\right)$ and $\left(m^{\circ}, b_{1}^{\circ}, \ldots, b_{j}^{\circ}\right)$ (resp.). $b_{\sigma j}^{\circ}$ constitute a solution of the following linear equations in $b_{\sigma j}$,

$$
I_{j}\left(m^{\circ}, b_{1}^{\circ}, \ldots, b_{j-1}^{\circ}, b_{j}\right)=0
$$

whose rank is $p+q-r_{j}^{\circ}$. Therefore it is always possible to choose a subset $J \subset\{n+1, \ldots, n+p+q\}$ consisting of $r_{j}^{\circ}$ distinct members such that (5.19) together with

$$
b_{\lambda j}-b_{\lambda j}^{\circ}=0, \quad \lambda \in J,
$$

form a compatible linear system of rank $p+q$. In other words, if $F$ is the $\left(p+q+j-r_{j}^{\circ}\right)$-dim submanifold defined in a suitable neighborhood of $m^{\circ}$ in $\mathcal{W}_{j}\left(m^{\circ}\right)$ by

$$
z_{\lambda}-g_{\lambda}\left(x_{1}, \cdots, x_{j}\right)=0, \quad \lambda \in J
$$

then we have

$$
F \supset N_{j} \supset N_{j-1}
$$

and since $T_{m} \mathrm{~d}(F)$ as a subspace of $T_{m} \mathrm{~d}\left(D_{j}\right)$ is defined by the equations $d z_{\lambda}-$ $\Sigma_{\mu=1} b_{\lambda \mu}^{\circ} d x_{\mu}=0$, we have also

$$
T_{m} \circ(F) \cap H\left(T_{m} \circ\left(N_{j-1}\right), R_{j} I\left(m^{\circ}\right)\right)=T_{m} \circ\left(N_{j}\right) .
$$

Associated with $F$ we will construct a submanifold $\tilde{F}$ of $\mathscr{U}_{j} \times \mathbf{R}^{p} \times(-1,1)$. Since all the functions under consideration are real analytic, by writing down appropriate convergent power series explicitly or otherwise, we can always find analytic functions $G_{\lambda}$ defined on a neighborhood of $(0, \ldots, 0 ; 0)$ in $\mathbf{R}^{j} \times$ $(-1,1)$ such that

$$
\begin{aligned}
G_{\lambda}\left(x_{1}, \ldots, x_{j-1}, 0 ; t\right) & =f_{\lambda}\left(x_{1}, \ldots, x_{j-1} ; t\right), \quad \lambda \in J . \\
\left(\partial G_{\lambda} / \partial t\right)\left(x_{1}, \cdots, x_{j} ; 0\right) & =\tau_{\lambda}\left(x_{1}, \cdots, x_{j}\right),
\end{aligned}
$$


Then $\tilde{F}$ is the $\left(2 p+q+j+1-r^{\circ}\right)$-dim submanifold in a neighborhood of $\tilde{m}^{\circ}$ in $\tau_{j} \times$ $\mathbf{R}^{p} \times(-1,1)$ which is defined by

$$
z_{\lambda}-G_{\lambda}\left(x_{1}, \cdots, x_{j} ; t\right)=0, \quad \lambda \in J .
$$

It follows from $(5.24)$ that $\pi_{1 *}\left(T_{\tilde{m}} \mathrm{~d}(\tilde{F})\right)=T_{m} d(F)$. If $\widetilde{E}_{j+1}$ is any $(j+1)$-dim integral element of $\mathcal{R}_{j} \tilde{I}\left(m^{\circ}\right)$ such that $T_{m} \propto(\tilde{F}) \supset \widetilde{E}_{j+1} \supset \widetilde{E}_{j}^{j+1}$, then we have

$$
T_{m \circ}(F) \supset \pi_{1 *}\left(\tilde{E}_{j+1}\right) \supset \pi_{1 *}\left(\tilde{E}_{j}\right)=T_{m o}\left(N_{j-1}\right) .
$$

But $\pi_{1 *}\left(\tilde{E}_{j+1}\right) \subset H\left(T_{m} d\left(N_{j-1}\right), R_{j} I\left(m^{\circ}\right)\right)$, therefore by $(5.23)$ we have $\pi_{1 *}\left(\tilde{E}_{j+1}\right)=$ $T_{m} \mathrm{~d}\left(N_{j}\right)$. However, we know by Remark 4.1 that such an $\widetilde{E}_{j+1}$ always exists and is unique. That is, $T \tilde{m}^{\circ}(\tilde{F}) \cap H\left(T \tilde{m}^{\circ}\left(\tilde{N}_{j-1}\right), \mathcal{R}_{j} \tilde{I}\left(m^{\circ}\right)\right)=\widetilde{E}_{j+1}$. By Theorem 1.2 , there exists in a neighborhood $\widetilde{U}_{j} \subset \mathbb{\sim}_{j} \times \mathbf{R}^{p} \times(1,1)$ of $\tilde{m}^{\circ}$ a $(j+1)$-dim integral manifold $\tilde{N}_{j}$ of $\mathscr{R}_{j} \tilde{I}(m)$ such that $F \cap \tilde{U}_{j} \supset \tilde{N}_{j} \supset \tilde{N}_{j-1} \cap \widetilde{U}_{j}$. We can assume that $\tilde{N}_{j} \cap \tilde{U}_{j}$ is defined by

$$
\begin{aligned}
& z_{\sigma}=b_{\sigma}\left(x_{1}, \cdots, x_{j} ; t\right), \\
& \xi_{\alpha}=\zeta_{\alpha}\left(x_{1}, \cdots, x_{j} ; t\right) .
\end{aligned}
$$

Then, in a suitable neighborhood of $(0, \ldots, 0 ; 0) \times \mathbf{R}^{j-1} \times(-1,1)$, we have

$$
\begin{aligned}
b_{\sigma}\left(x_{1}, \cdots, x_{j-1}, 0 ; t\right) & =f_{\sigma}\left(x_{1}, \cdots, x_{j-1} ; t\right), \\
b_{\lambda}\left(x_{1}, \cdots, x_{j} ; t\right) & =G_{\lambda}\left(x_{1}, \cdots, x_{j} ; t\right) .
\end{aligned}
$$

Let ${ }^{t} N_{j}$ be the one-parameter family of integral manifolds of $\mathcal{R}_{j} I(\mathrm{~m} g)$ defined by (5.27).

If we put

$$
z_{\sigma}=b_{\sigma}\left(x_{1}, \cdots, x_{j} ; 0\right), \quad \xi_{\sigma}=\left(\partial b_{\sigma} / \partial t\right)\left(x_{1}, \cdots, x_{j} ; 0\right),
$$

then, in a neighborhood of $m^{\prime 0}$ in $W_{j} \times \mathbf{R}^{p+q},(5.30)$ defines a $j$-dim integral manifold $\pi_{j}^{\prime}$ of $R_{j} I^{\prime}\left(m^{\circ}\right)$. The restriction of $\pi_{j}^{\prime}$ to $\mho_{j-1} \times \mathbf{R}^{p+q}$ defines a $(j-1)$ dim integral manifold $N_{j-1}^{\prime}$ of $\Re_{j-1} I^{\prime}\left(m^{\circ}\right)$, consequently also of $R_{j} I^{\prime}(m 9)$. We put $E_{j-1}^{\prime}=T_{m} \prime d\left(N_{j-1}^{\prime}\right)$. Since $\pi_{2 *}\left(E_{j-1}^{\prime}\right)=T_{m} \mathrm{~d}\left(N_{j-1}\right)$ is a regular integral element of $\Re_{j} I\left(m^{\circ}\right)$, it follows readily from $\mathbf{P}_{j}^{1}$ that $E_{j-1}^{\prime}$ is also a regular integral element of $\mathcal{R}_{j} I^{\prime}(\mathrm{m} 9$ and

$$
r_{j}\left(E_{j-1}^{\prime \circ}, R_{j} I^{\prime}\left(m^{\circ}\right)\right)=2 r_{j}^{\circ}
$$

Let $F^{\prime}$ be the $\left(2(p+q)+j-2 r_{j}^{9}\right)$-dim submanifold in a neighborhood of $m^{\prime \circ}$ in $w_{j} \times \mathbf{R}^{p+q}$ which is defined by the following equations:

$$
\begin{aligned}
z_{\lambda}-G_{\lambda}\left(x_{1}, \cdots, x_{j} ; 0\right) & =0, \\
\xi_{\lambda}-\left(\partial G_{\lambda} / \partial t\right)\left(x_{1}, \ldots, x_{j} ; 0\right) & =0,
\end{aligned}
$$

Then in a suitable neighborhood $\mathcal{U}_{j}^{\prime} \subset \mathcal{W}_{j} \times \mathbf{R}^{p+q}$ of $m^{\prime \circ}$ we have (by (5.13) and (5.29)) 


$$
\begin{aligned}
& F^{\prime} \cap \mathcal{U}_{j}^{\prime} \supset \Re_{j}^{\prime} \cap \mathcal{U}_{j}^{\prime} \supset N_{j-1}^{\prime} \cap \mathcal{U}_{j}^{\prime}, \\
& F^{\prime} \cap \mathcal{U}_{j}^{\prime} \supset N_{j}^{\prime} \cap \mathcal{U}_{j}^{\prime} \supset N_{j-1}^{\prime} \cap \mathcal{U}_{j}^{\prime} .
\end{aligned}
$$

Since $\pi_{2}\left(F^{\prime}\right)=F$ and $\pi_{2}\left(N_{j-1}^{\prime}\right)=N_{j-1}, .(5.23)$ and the uniqueness assertion of Theorem 1.2 imply that $\pi_{2}\left(\mathcal{\gamma}_{j}^{\prime}\right)=\pi_{2}\left(N_{j}^{\prime}\right)$. That is,

$$
b_{\sigma}\left(x_{1}, \cdots, x_{j} ; 0\right)=g_{\sigma}\left(x_{1}, \cdots, x_{j} ; 0\right)
$$

in a suitable neighborhood of $(0, \ldots: ; 0) \times \mathbf{R}^{j}$.

Now if we put

$$
a_{\sigma \kappa}^{\circ}=\left(\partial^{2} b_{\sigma} / \partial t \partial x_{\kappa}\right)(0, \ldots ; 0) \quad(1 \leq \kappa \leq j)
$$

then $T_{m^{\prime}} \mathrm{O}\left(F^{\prime}\right)$ as a subspace of $T_{m^{\prime}} \mathrm{d}\left(C_{j} \times \mathbf{R}^{p+q}\right)$ is defined by the equations

$$
d z_{\lambda}-\sum_{\kappa=1}^{j} b_{\lambda \kappa}^{0} d x_{\kappa}=0,
$$

$$
d \xi_{\lambda}-\sum_{\kappa=1}^{j} a_{\lambda \kappa}^{0} d x_{\kappa}=0 .
$$

$\lambda \in J$

Let $b_{\kappa}^{\prime \circ}$ be the vector whose components are $b_{\sigma K}^{\circ}, a_{\sigma K^{\circ}}^{\circ}$. Then $E_{j-1}^{\prime}$ and $T_{m^{\prime} \circ}\left(\eta_{j}^{\prime}\right)$ as integral elements of $\mathcal{R}_{j=1} I^{\prime}\left(m^{\circ}\right)$ and $\Re_{j} I^{\prime}\left(m^{\circ}\right)$ (resp.) are defined by ( $m^{\prime 0}$, $\left.b_{1}^{\prime \circ}, \ldots, b_{j-1}^{\prime \circ}\right)$ and $\left(m^{\prime \circ}, b_{1}^{\prime \circ}, \cdots, b_{j}^{\prime \circ}\right)$ (resp.). $b_{j}^{\prime \circ}$ are solutions of the following system of equations in $b_{j}^{\prime}$ :

$$
\begin{aligned}
& I_{j}^{\prime}\left(m^{\prime \circ}, b_{1}^{\circ \circ}, \cdots, b_{j-1}^{\circ \circ}, b_{j}^{\prime}\right)=0, \\
& b_{\lambda j}-b_{\lambda j}^{\circ}=0, \quad \lambda \in J, \\
& a_{\lambda j}-a_{\lambda j}^{\circ}=0 . \quad \lambda \in J .
\end{aligned}
$$

Since $E_{j-1}^{\prime}$ is a regular integral element of $\mathcal{R}_{j} I^{\prime}\left(m^{\circ}\right)$, it follows from (5.31) that (5.37) is a compatible system of rank $2(p+q)-2 r_{j}^{\circ}$. Denote by $\left(4.17_{j}\right)^{*}$ and $\left(4.18_{j}\right)^{*}$ the linear equations in $b_{j}^{\prime}$ obtained by setting $b_{\sigma \mu}=b_{\sigma \mu}^{\circ}, a_{\sigma \mu}=a_{\sigma \mu}^{\circ}$, $\xi_{\sigma}=\xi_{\sigma}^{\circ}$ and $m=m^{\circ}$ in $\left(4.17_{j}\right)$ and $\left(4.18_{j}\right)$ (resp.). Then $(5.37)$ is generated by $\left(4.17_{j}\right)^{*}$ and $\left(4.18_{j}\right)^{*}$. $\left(4.17_{j}\right)^{*}$ also generates (5.19). By Remark 4.4 we know that the coefficient matrix of $b_{\sigma_{j}}$ in $\left(4.17_{j}\right)^{*}$ is the same as that of $a_{\sigma_{j}}$ in $\left(4.18_{j}\right)^{*}$. Now, by our choice of the subset $J,(5.19)$ and (5.20) have rank $p+q$ as linear equations in $b_{\sigma j}$. Therefore $\left(4.18_{j}\right)^{*}$ and (5.38) have rank $p+q$ as linear equations in $a_{\sigma j}$. In other words, (5.37), (5.20) and (5.38) have rank $2(p+q)$ as linear equations in $b_{j}^{\prime}$ (maximal rank). That is,

$$
T_{m^{\prime} \mathrm{d}}\left(F^{\prime}\right) \cap H\left(E_{j-1}^{\prime}, \mathbb{R}_{j} I^{\prime}\left(m^{\circ}\right)\right)=T_{m^{\prime}}\left(\Re_{j}^{\prime}\right)
$$


Since $T_{m^{\prime}} \circ\left(N_{j}^{\prime}\right) \subset T_{m^{\prime}} \circ\left(F^{\prime}\right) \cap H\left(E_{j-1}^{\prime}, R_{j} I^{\prime}\left(m^{\circ}\right)\right)$, therefore $T_{m^{\prime 0}}\left(N_{j}^{\prime}\right)=T_{m^{\prime 0}}\left(\Re_{j}^{\prime}\right)$. By Theorem 1.1 and (5.33) we can conclude that $N_{j}^{\prime} \cap \mathcal{U}_{j}^{\prime}=\pi_{j}^{\prime} \cap \mathcal{U}_{j}^{\prime}$. In particular, this implies that the restriction of the $R_{j} I(m)$-field $\mathbf{v}$ to $N_{j} \cap \pi_{2}\left(\mathcal{U}_{j}^{\prime}\right)$ coincides with the restriction of the deformation vector of the one-parameter family of integral manifolds ${ }^{t} N_{j}$ on ${ }^{0} N_{j}$ to ${ }^{0} N_{j} \cap \pi_{2}\left(\mathcal{U}_{j}^{\prime}\right)$.

Proof of (c). Let

$$
\left\{m^{\circ}\right\}=E_{0} \subset E_{1} \subset \cdots \subset E_{j} \subset E_{j+1}
$$

be a regular integral chain of $\mathscr{R}_{j+1} I\left(m^{9}\right)$ such that $d x_{1} \wedge \ldots \wedge d x_{\mu} \neq 0$ on $E_{\mu}$ $(1 \leq \mu \leq j+1)$ and $m^{\prime \circ}=\left(m^{\circ}, \xi_{\sigma}^{\circ}\right) \in \mathbb{W}_{j+1}\left(m^{\circ}\right) \times \mathbf{R}^{p+q}$. Let

$$
\left\{m^{\prime \circ}\right\}=E_{0}^{\prime} \subset E_{1}^{\prime} \subset \cdots \subset E_{j-1}^{\prime} \subset E_{j}^{\prime}
$$

be an integral chain of $\mathcal{R}_{j} I^{\prime}\left(m^{\circ}\right)$ such that

$$
\pi_{2 *}\left(E_{\nu}^{\prime}\right)=E_{\nu} \quad(0 \leq \nu \leq j)
$$

By $\mathbf{P}_{j}^{1}$ such integral chain always exists and furthermore it is also regular. Applying Theorem 1.2, we can find a general solution $N_{j}^{\prime}$ of $R_{j} I^{\prime}\left(m^{\circ}\right)$ through $m^{\prime \circ}$ such that $T_{m^{\prime}} \circ\left(N_{j}^{\prime}\right)=E_{j}^{\prime}$. Restricted to a subset of $N_{j}^{\prime}$, if necessary, this will give us a normal solution $\pi_{2}\left(N_{j}^{\prime}\right)=N_{j}$ of $\mathcal{R}_{j} I\left(m^{\circ}\right)$ with an $\mathcal{R}_{j} I\left(m^{\circ}\right)$-field $v$ on it which can be defined in a neighborhood $m^{\circ}$ by

$$
\begin{aligned}
z_{\sigma} & =g_{\sigma}\left(x_{1}, \ldots, x_{j}\right), \\
\mathbf{v}(m) & =\sum_{\sigma} \tau_{\sigma}\left(x_{1}(m), \cdots, x_{j}(m)\right)\left(\frac{\partial}{\partial z_{\sigma}}\right)_{m} .
\end{aligned}
$$

We may assume that $x_{1}\left(m^{\circ}\right)=\cdots=x_{j}\left(m^{9}\right)=0$. (5.43) is a set of normal data of $\Re_{j} I\left(m^{\circ}\right)$. Applying $\mathbf{P}_{j}^{2}$ we can construct a one-parameter family of integral manifolds ${ }^{t} N_{j}\left(t \in\left(-\epsilon_{j}, \epsilon_{j}\right)\right.$ and $\left.\epsilon_{j}>0\right)$ of $R_{j} I(m 9)$ in a neighborhood of $m^{\circ}$ in $\mathcal{C}_{j}(m)$ which can be defined as

$$
z_{\sigma}=f_{\sigma}\left(x_{1}, \cdots, x_{j} ; t\right)
$$

and such that

$$
\begin{aligned}
f_{\sigma}\left(x_{1}, \ldots, x_{j} ; 0\right) & =g_{\sigma}\left(x_{1}, \ldots, x_{j}\right), \\
\left(\partial f_{\sigma} / \partial t\right)\left(x_{1}, \ldots, x_{j} ; 0\right) & =\tau_{\sigma}\left(x_{1}, \ldots, x_{j}\right) .
\end{aligned}
$$

We put

$$
\begin{aligned}
& k_{\sigma \kappa}(t)=\left(\partial f_{\sigma} / \partial x_{\kappa}\right)(0, \cdots, 0 ; t) \\
& a_{\sigma \kappa}(t)=\left(\partial^{2} f_{\sigma} / \partial t \partial x_{\kappa}\right)(0, \cdots, 0 ; t) \quad(1 \leq \kappa \leq j) .
\end{aligned}
$$

Denote by $b_{\kappa}^{\prime}(t)$ the vector whose components are $b_{\sigma \kappa}(t), a_{\sigma \kappa}(t)$ and also by $b_{K}(t)$ the vector whose components are $b_{\sigma K}(t)$. Let $c:\left(-\epsilon_{j}, \epsilon_{j}\right) \rightarrow \ell_{j}\left(m^{\circ}\right)$ be the 
analytic mapping such that for every fixed $t \in\left(-\epsilon_{j}, \epsilon_{j}\right)$ the coordinates of $c(t)$ are given by

$$
\begin{aligned}
x_{\kappa} & =0 \\
z_{\sigma} & =f_{\sigma}(0, \ldots, 0 ; t) \quad(1 \leq \kappa \leq j)
\end{aligned}
$$

$E^{\prime}{ }_{j}$ and $E_{j}$ as integral elements of $\mathcal{R}_{j} I^{\prime}\left(m^{\circ}\right)$ and $\mathcal{R}_{j} I\left(m^{\circ}\right)$ (resp.) are defined by $\left(m^{\prime \circ}, b_{1}^{\prime}(0), \ldots, b_{j}^{\prime}(0)\right)$ and $\left(m^{\circ}, b_{1}(0), \ldots, b_{j}(0)\right)$ (resp.). $T_{c(t)}\left({ }^{t} N_{j}\right)$ as an integral element of $\Re_{j} I(m)$ is defined by $\left(c(t), b_{1}(t), \ldots, b_{j}(t)\right)$. Let $\left(4.17_{j+1}\right)^{t}$ be the system of linear equations in $b_{\sigma, j+1}$ obtained by setting $b_{\sigma \mu}=b_{\sigma \mu}(t)$ and $m=c(t)$ in $\left(4.17_{j+1}\right)$. Also let $\left(4.18_{j+1}\right)^{0}$ be the linear system in $b_{j+1}^{\prime}$ obtained by setting $b_{\sigma \mu}=b_{\sigma \mu}(0), a_{\sigma \mu}=a_{\sigma \mu}(0), \xi_{\sigma}=\xi_{\sigma}^{\circ}$ and $m=c(0)$ in $\left(4.18_{j+1}\right)$. Then it is clear that $\left(4.17_{j+1}\right)^{t}$ generates $I_{j+1}\left(c(t), b_{1}(t), \ldots, b_{j}(t), b_{j+1}\right)$, $\left(4.17_{j+1}\right)^{0}$ and $\left(4.18_{j+1}\right)^{0}$ generate $I_{j+1}^{\prime}\left(m^{\prime \circ}, b_{1}^{\prime}(0), \ldots, b_{j}^{\prime}(0), b_{j+1}^{\prime}\right)$. Since $E_{j}$ is a regular integral element of $\mathscr{R}_{j+1}^{+1} I\left(m^{\circ}\right),\left(4.17_{j+1}\right)^{0}$ is a compatible linear system of rank $p+q-r_{j+1}^{\circ}$, where $r_{j+1}^{\circ}=r_{j+1}\left(E_{j}, R_{j+1}^{j+1} I(m g)\right)$. Since $T_{c(0)}\left({ }^{0} N_{j}\right)$ $=E_{j}$, for $t$ sufficiently close to 0 , say $|t|<\epsilon, T_{c(t)}\left({ }^{t} N_{j}\right)$ is also a regular integral element (see Remark 1.1) and $\left(4.17_{j+1}\right)^{t}$ is also of rank $p+q-r_{j+1}^{\circ}$. For every fixed $|t|<\epsilon$, consider the following linear system in the variables $A_{\sigma, j+1}^{t}=b_{\sigma, j+1}+t a_{\sigma, j+1}$ :

$$
A_{a, j+1}^{t}+B_{a, j+1}(c(t))=0
$$

$(5.48)^{t} \sum_{\sigma}\left\{B_{\alpha \mu ; \sigma}(c(t)) A_{\sigma, j+1}^{t}-B_{\alpha, j+1 ; \sigma}(c(t)) b_{\sigma \mu}(t)\right\}$

$$
+B_{a \mu ; j+1}(c(t))-B_{a, j+1 ; \mu}(c(t))=0 \quad(1 \leq \mu \leq j)
$$

( $B_{a_{k}}$ and $B_{\alpha_{k} ; \sigma}$ are the same functions as in previous section.) Note that $(5.48)^{t}$ is obtained by setting $b_{\sigma, j+1}=A_{\sigma, j+1}^{t}$ in $(4.17)^{t}$. Therefore $(5.48)^{t}$ is a compatible linear system of rank $p+q-r_{j+1}^{\circ}$. It is important to observe that if we differentiate each equation of $(5.48)^{t}$ with respect to $t$ at $t=0$ and making use of the facts that $d b_{\alpha \mu} / d t=a_{a \mu}$ and $B_{a \mu ; \sigma ; \rho}=B_{a \mu ; \rho ; \sigma}$, we will have precisely $\left(4.18_{j+1}\right)^{0}$. For $t$ of sufficiently small absolute value, there is an obvious one-to-one correspondence between $\left(4.17_{j+1}\right)^{0}$ and $(5.48)^{t}$ which preserves generators as linear equations in $b_{\sigma, j+1}$ and $A_{\sigma_{0, j+1}}^{t}$ respectively. Denote such a correspondence by $\mathcal{H}_{t}:\left(4.17_{j+1}\right)^{0} \rightarrow(5.48)^{t}$. For $L \in\left(4.17_{j+1}\right)^{0}$, $\mathcal{H}_{t}(L)$ depends analytically on $t$. Define a one-to-one correspondence $\delta$ : $\left(4.17_{j+1}\right)^{0} \rightarrow\left(4.18_{j+1}\right)^{0}$ by

$$
\delta(L)=\partial \mathcal{H}_{t}(L) /\left.\partial t\right|_{t=0} \text { for } L \in\left(4.17_{j+1}\right)^{0}
$$


Let $S=\left\{L_{1}, \ldots, L_{a}\right\}$ be a set of linearly independent generators of $\left(4.17_{j+1}\right)^{0}$. We claim that $S \cup \delta(S)$ is a set of generators for the linear system $\left(4.17_{j+1}\right)^{0} \cup$ $\left(4.18_{j+1}\right)^{0}$. Indeed, let $\delta(L) \in\left(4.18_{j+1}\right)^{0}$, then there exist functions $\phi_{1}(t), \ldots$, $\phi_{a}(t)$ defined for $t$ sufficiently close to 0 such that

$$
\mathcal{H}_{t}(L)=\phi_{1}(t) \mathcal{H}_{t}\left(L_{1}\right)+\cdots+\phi_{a}(t) \mathcal{H}_{t}\left(L_{a}\right) .
$$

Using elementary linear algebra and the fact that $\mathcal{H}_{t}\left(L_{1}\right), \ldots, \mathcal{H}_{t}\left(L_{a}\right)$ as well as $\mathcal{H}_{t}(L)$ depend analytically on $t$, one can easily show that $\phi_{1}(t), \ldots, \phi_{a}(t)$ are actually analytic functions of $t$. Therefore we have

$$
\begin{aligned}
\delta(L) & =\partial \mathcal{H}_{t}(L) /\left.\partial t\right|_{t=0} \\
& =\phi_{1}^{\prime}(0) L_{1}+\cdots+\phi_{a}^{\prime}(0) L_{a}+\phi_{1}(0) \delta\left(L_{1}\right)+\cdots+\phi_{a}(0) \delta\left(L_{a}\right) .
\end{aligned}
$$

Therefore there are at most $2\left(p+q-r_{j+1}^{\circ}\right)$ linear independent equations in the linear system $\left(4.17_{j+1}\right)^{0} \cup\left(4.18_{j+1}\right)^{0}$. However, it follows from Remark 4.4 that the coefficient matrix of $a_{\sigma, j+1}$ in $\left(4.18_{j+1}\right)^{0}$ is exactly the same as that of $b_{\sigma, j+2}$ in $\left(4.17_{j+1}\right)^{0}$. Therefore $\left(4.17_{j+1}\right)^{0} \cup\left(4.18_{j+1}\right)^{0}$ is a compatible linear system in the variables $a_{\sigma, j+1}, b_{\sigma, j+1}$ and it is of rank $2\left(p+q-r_{j+1}^{\circ}\right)$. Thus we can always find a $(j+1)$-dim integral element $E_{j+1}^{\prime}$ of $R_{j+1} I^{\prime}\left(m^{\circ}\right)$ such that $E_{j+1}^{\prime} \supset E_{j}^{\prime}$ and $\pi_{2 *}\left(E_{j+1}^{\prime}\right)=E_{j+1}$. We have also proved that $r_{j+1}\left(E_{j}^{\prime}, \mathcal{R}_{j+1} I^{\prime}\left(m^{\circ}\right)\right)=$ ${ }_{2 r}{ }_{j+1}\left(E_{j}, \Re_{j+1} I\left(m^{\circ}\right)\right)$. Now if $E_{j}^{\prime *}$ is a $j$-dim integral element of $\Re_{j} I^{\prime}\left(m^{\circ}\right)$ defined by $\left(m^{\prime *}, b_{1}^{\prime *}, \ldots, b_{j}^{\prime *}\right)$ in a sufficiently small neighborhood of $\left(m^{\prime 0}, b_{j}^{\prime}(0), \ldots\right.$, $\left.b_{j}^{\prime}(0)\right)$, we can construct an integral chain of $\left.\mathcal{R}_{j} I^{\prime}\left(m^{\circ}\right)\right)$ using subspaces $E_{\mu}^{\prime *}$ of $E_{j}^{\prime *}$ on which $d x_{\mu+1}=\cdots=d x_{j}=0(1 \leq \mu \leq j)$. Under $\pi_{2 *}$ this will be projected onto an integral chain of $\mathscr{R}_{j} I\left(m^{\circ}\right)$ which is also regular, since it is 'close' to the regular integral chain (5.40). Repeating the above argument, we can prove that $I_{j+1}^{\prime}\left(m^{\prime *}, b_{j}^{\prime *}, \ldots, b_{j}^{\prime *}, b_{j+1}^{\prime}\right)$ is a compatible linear system in $b_{j+1}^{\prime}$ and

$$
\begin{aligned}
r_{j+1}\left(E_{j}^{\prime *}, \mathcal{R}_{j+1} I^{\prime}\left(m^{\circ}\right)\right) & =2 r_{j+1}\left(\pi_{2 *}\left(E_{j}^{\prime *}\right), \mathcal{R}_{j+1} I\left(m^{\circ}\right)\right) \\
& =2 r_{j+1}\left(E_{j}, \mathcal{R}_{j+1} I\left(m^{\circ}\right)\right)=r_{j+1}\left(E_{j}^{\prime}, \mathbb{R}_{j+1} I^{\prime}\left(m^{\circ}\right)\right) .
\end{aligned}
$$

The second equality follows since $\pi_{2 *}\left(E_{j}^{\prime *}\right)$ is 'close' to $E_{j}$. We have thus proved that we can extend (5.40) to an integral chain of $R_{j+1}^{j} I^{\prime}\left(m^{\circ}\right)$ and any such extension is also regular. This completes the proof of Proposition 5.1.

6. Applications to Jacobi fields on minimal submanifolds. Let $M$ be a Riemannian manifold of dimension $n+p$ and $F(M)$ be the bundle of orthonormal frames of $M$ with bundle projection $\pi: F(M) \rightarrow M$. Let $\omega_{A}$ be the solder 1 -forms and $\omega_{A B}$ be the 1-forms which define the Riemannian connection of $F(M)$ (see for example [1]). The local geometry of $M$ is completely determined by the following structure equations, 


$$
\begin{aligned}
d \omega_{A}=\sum_{B} \omega_{B} \wedge \omega_{B A}, & \omega_{A B}+\omega_{B A}=0, \\
d \omega_{A B}=\sum_{C} \omega_{A C} \wedge \omega_{C B}+\Omega_{A B}, & \Omega_{A B}=-\frac{1}{2} \sum_{C, D} R_{A B C D} \omega_{C} \wedge \omega_{D} .
\end{aligned}
$$

Let $I$ be the ideal in the ring of analytic differential forms on $F(M)$ which is generated by the following forms,

$$
\omega_{\alpha}, \quad \sum_{i} \omega_{i} \wedge \omega_{i \alpha}
$$

$$
\Theta_{\alpha} \equiv \sum_{i} \omega_{1} \wedge \cdots \wedge \omega_{i-1} \wedge \omega_{i a} \wedge \omega_{i+1} \wedge \cdots \wedge \omega_{n}
$$

It is easy to see, using (6.1), that $d I \subset I$. Therefore, $I$ defines an exterior differential system on $F(M)$.

Proposition 6.1. I is involutive with independent variables $\left\{\omega_{1}, \ldots, \omega_{n-1}\right.$, $\left.\omega_{12}, \cdots, \omega_{n-1, n}, \omega_{n+1, n+2}, \cdots, \omega_{n+p-1, n+p}, \omega_{n}\right\}$ at every point $e \in F(M)$ $\left(\left\{\omega_{12}, \cdots, \omega_{n-1, n}\right\}\right.$ and $\left\{\omega_{n+1, n+2}, \cdots, \omega_{n+p-1, n+p}\right\}$ are some orderings of the sets $\left\{\omega_{i j} ; i<j\right\}$ and $\left\{\omega_{\alpha \beta} ; \alpha<\beta\right\}$ respectively).

Proof. We put

$$
\omega_{\alpha}=\sum_{i} a_{\alpha_{i} \omega_{i}}+\sum_{i<j} a_{\alpha_{i j}} \omega_{i j}+\sum_{\beta<\gamma} a_{\alpha \beta \gamma} \omega_{\beta \gamma},
$$

$$
\omega_{i a}=\sum_{j} b_{i \alpha_{j}} \omega_{j}+\sum_{j<k} b_{i \alpha_{j k}} \omega_{j k}+\sum_{\beta<\gamma} b_{i \alpha \beta \gamma} \omega_{\beta \gamma} .
$$

Let $b_{i}$ be the vector whose components are $a_{\gamma i}, b_{k \gamma i}$. Also let $b_{i j^{\prime}} i<j$ $\left(b_{\alpha \beta}, \alpha<\beta\right)$ be the vector whose components are $a_{\gamma i j}, b_{k \gamma i j}\left(a_{\gamma \alpha \beta}, b_{k \gamma \alpha \beta}\right)$. Also put

$$
\lambda=n(n-1) / 2+p(p-1) / 2 .
$$

We will find, for $1 \leq \mu \leq n-1$, that the equations $I_{\mu}\left(e, b_{1}, \ldots, b_{\mu}\right)=0$, after taking into account that $I_{\mu-1}\left(e, b_{1}, \ldots, b_{\mu-1}\right)=0$ is generated by

$$
a_{\alpha \mu}=0, \quad b_{\nu \alpha \mu}-b_{\mu \alpha \nu}=0 \quad(1 \leq \nu \leq \mu-1) .
$$

It is also not difficult to see that there is only one way to extend any system of solutions of the equations $I_{n-1}\left(e, b_{1}, \ldots, b_{n-1}\right)=0$ to a system of solutions of the equations

$$
I_{n+\lambda-1}\left(e, b_{1}, \cdots, b_{n-1}, b_{12}, \cdots, b_{n, n-1} .\right.
$$

$$
\left.b_{n+1, n+2}, \cdots, b_{n+p-1, n+p}\right)=0 \text {, }
$$


namely, by setting

$$
b_{i j}=b_{a \beta}=0 \text {. }
$$

After taking account of the equations

$$
I_{n+\lambda-i}\left(e, b_{1}, \cdots, b_{n-1}, b_{12}, \cdots, b_{n+p-1, n+p}\right)=0 \text {, }
$$

the equations $I_{n+\lambda}\left(e, b_{1}, \ldots, b_{n-1}, b_{12}, \ldots, b_{n+p-1, n+p}, b_{n}\right)=0$ are generated by

$$
\begin{aligned}
a_{a n} & =0, \\
b_{\mu a n}-b_{n a \mu} & =0 \quad(1 \leq \mu \leq n-1), \\
b_{n a n}+\sum_{\mu=1}^{n-1} b_{\mu a \mu} & =0 .
\end{aligned}
$$

It follows that any system of solutions $\left(e^{\circ}, b_{1}^{\circ}, \ldots, b_{n-1}^{\circ}, b_{12}^{\circ}, \ldots, b_{n+p-1, n+p^{\circ}}^{\circ}\right.$ $\left.b_{n}^{\circ}\right)$ of (6.5), (6.7) and (6.9) defines a regular integral flag $\left\{e^{\circ}\right\}=E_{0} \subset E_{1} \subset \ldots \subset$ $E_{n+\lambda-1} \subset E_{n+\lambda}$ of $I$. It is also easy to see that

$$
r_{\lambda+n}\left(E_{\lambda+n-1}, I\right)=0 \text {. Q.E.D. }
$$

Remark 6.1. (6.10) implies that through any $(\lambda+n-1)$-dim integral element $E_{\lambda+n-1}$ of $I$ on which

$$
\omega_{1} \wedge \ldots \wedge \omega_{n-1} \wedge \omega_{12} \wedge \cdots
$$

$$
\wedge \omega_{n-1, n} \wedge \omega_{n+1, n+2} \wedge \cdots \wedge \omega_{n+p-1, n+p} \neq 0
$$

there exists a unique $(\lambda+n)$-dim integral element $E_{\lambda+n}$ of $I$ on which

$$
\omega_{1} \wedge \cdots \wedge \omega_{n-1} \wedge \omega_{12} \wedge \cdots
$$

$$
\wedge \omega_{n-1, n} \wedge \omega_{n+1, n+2} \wedge \cdots \wedge \omega_{n+p-1, n+p} \wedge \omega_{n} \neq 0
$$

and $E_{\lambda+n} \supset E_{\lambda+n-1}$.

Remark 6.2. We have proved above that any integral manifold of $I$ on which (6.11) holds is a general solution of $I$.

If $f: F^{a} \rightarrow F(M)$ defines an integral manifold of $I$ on which (6.12) holds, then $\operatorname{dim} F^{a}=n(n-1) / 2+p(p-1) / 2+n(=\lambda+n) . \quad f\left(F^{a}\right)$ is the bundle space of the bundle of adapted frames (see for example [1]) of the submanifold $\pi \circ f\left(F^{a}\right.$ ) of $M$. In fact, $\pi \circ f\left(F^{a}\right)$ is an $n$-dim minimal submanifold of $M$ since the pullbacks of $\Theta_{a}$ through any section of the adapted frame bundle are the mean curvature forms of $\pi \circ f\left(F^{a}\right)$ (see for example [4]). Conversely, given any $n$-dim minimal submanifold $N$ of $M$, the bundle of adapted frames over $N$ considered as a submanifold of $F(M)$ is a general solution of $I$. The involutiveness of $I$ implies the local existence of minimal submanifolds of dimension $n$. More precisely, we have the following theorem (see also [10] and [12]). 
Theorem 6.1. Let $G_{n-1}$ be an imbedded $(n-1)$-dim submanifold of $M$ and $P$ be an n-dim distribution along $G_{n-1}$ such that $T_{m}\left(G_{n-1}\right) \subset P(m)$ for all $m \epsilon$ $G_{n-1}$. Then assuming the data are real analytic, for eacb $m^{\circ} \in G_{n-1}$ there exists in every sufficiently small neighborbood $\mathcal{U}$ of $m^{\circ}$ a unique imbedded analytic minimal submanifold $N$ of dimension $n$ sucb that

1. $\mathcal{U} \supset N \supset G_{n-1} \cap \mathcal{U}$,

2. $T_{m}(N)=P(m)$ for all $m \in G_{n-1} \cap \mathcal{U}$.

Proof. Let $F^{!}\left(G_{n-1}\right)$ be the subset of $F(M)$ consisting of all frames $\left(m, e_{1}\right.$, $\cdots, e_{n+p}$ ) (i.e. $e_{1}, \ldots, e_{n+p}$ is an orthonormal basis of $T_{m}(M)$ ) such that $m \in G_{n-1}$ and $e_{1}, \ldots, e_{n}$ span $P(m)$. It can be easily seen that $F^{!}\left(G_{n-1}\right)$ is a submanifold of $F(M)$ of dimension $\lambda+n-1$. Indeed, if $G_{n-1}$ is defined by the imbedding $g: G_{n-1} \rightarrow M$, then $F^{!}\left(G_{n-1}\right)$ realizes a reduction of the group $O(n+p)$ of the induced bundle $g^{-1}(F(M))$ over $G_{n-1}$ to $O(n) \times O(p)$ (for definitions and facts related to induced bundle and reduction of structure group see for example [8]). Denote also by $\omega_{A}, \omega_{A B}$ their pullbacks to $F^{!}\left(G_{n-1}\right)$. Then, at every point of $F^{!}\left(G_{n-1}\right), \omega_{i j}, \omega_{\alpha \beta}$ and $\omega_{i}$ span the cotangent space of $F^{!}\left(G_{n-1}\right)$, but only $n-1$ of the $\omega_{i}$ are linearly independent while the $\omega_{i j}, \omega_{\alpha \beta}$ are independent among themselves and independent of the $\omega_{i}$. On $F^{!}\left(G_{n-1}\right)$ we have

$$
\omega_{\alpha}=0 \text {. }
$$

Taking the exterior derivative on both sides of (6.13), we have

$$
\sum_{i} \omega_{i} \wedge \omega_{i \alpha}=0
$$

This implies that, on $F^{!}\left(G_{n-1}\right), \omega_{i \alpha}$ are independent of $\omega_{i j}, \omega_{\alpha \beta}$ and they are only linear combinations of the $\omega_{i}$. Since on $F^{!}\left(G_{n-1}\right)$ only $n-1$ of the $\omega_{i}$ are linear independent, the restriction of $\Theta_{\alpha}$ (in (6.2)) to $F^{!}\left(G_{n-1}\right)$ vanishes identically. $F^{!}\left(G_{n-1}\right)$ is therefore a $(\lambda+n-1)$-dim integral manifold of $I$ on which (6.11) holds. By Remark 6.1, we have that at every point $e^{\circ} \in F^{!}\left(G_{n-1}\right)$ there exists a unique $(\lambda+n)$-dim integral element $E_{\lambda+n}$ of $I$ on which (6.12) holds and $E_{\lambda+n} \supset T_{e^{\circ}}\left(F^{!}\left(G_{n-1}\right)\right)$. Since $r_{\lambda+n}\left(T_{e^{\circ}}\left(G_{n-1}\right), I\right)=0$, we have by Theorem 1.1 that there exists, in a sufficiently small neighborhood $\tilde{U}$ of $e^{\circ}$ in $F(M)$, a unique integral manifold $F^{a}$ of $I$ such that $T_{e^{o}}\left(F^{a}\right)=E_{\lambda+n}$ and $\tilde{U}$ $\supset F^{a} \supset F^{!}\left(G_{n-1}\right) \cap \tilde{U}$. Then, $\mathcal{U}=\pi(\widetilde{\mathcal{U}})$ is a neighborhood of $m^{\circ}=\pi\left(e^{\circ}\right)^{+n}$ and $N=$ $\pi\left(F^{a}\right)$ is a submanifold of $M$ which have the required properties. Q.E.D.

Let $f: F^{a} \rightarrow F(M)$ define a $(\lambda+n)$-dim integral manifold of $I$ on which (6.12) holds. We will now compute the equations of variation of $I$ on $f\left(F^{a}\right)$. Let $\tau: f\left(F^{a}\right) \rightarrow T(F(M))$ be an $I$-field defined on $f\left(F^{a}\right)$. For simplicity, we will assume that $0=\tau_{i}=\left\langle\tau, \omega_{i}\right\rangle$. In fact, only the components $\tau_{a}=\left\langle\tau, \omega_{a}\right\rangle, \tau_{i a}=$ $\left\langle\tau, \omega_{i \alpha}\right\rangle$ are of geometric significance. The equations of variation are 


$$
\left.\left.d f^{*}(\tau\lrcorner \omega_{a}\right)+f *(\tau\rfloor d \omega_{a}\right)=0,
$$

$$
\begin{aligned}
& \left.\left.d f *(\tau\lrcorner \sum_{i} \omega_{i} \wedge \omega_{i a}\right)+f^{*}(\tau\lrcorner d \sum_{i} \omega_{i} \wedge \omega_{i \alpha}\right)=0, \\
& \left.d f *\left(\tau \perp \Theta_{\alpha}\right)+f^{*}(\tau\lrcorner d \Theta_{\alpha}\right)=0 .
\end{aligned}
$$

Set $\theta_{A}=f^{*}\left(\omega_{A}\right), \theta_{A B}=f^{*}\left(\omega_{A B}\right)$. Considering $\tau_{\alpha}$ and $\tau_{i \alpha}$ as functions defined on $F^{a},(6.15)$ can be written as

$$
d \tau_{a}+\sum_{\beta} \tau_{\beta} \theta_{\beta a}-\sum_{i} \tau_{i a} \theta_{i}=0 .
$$

One will find (6.16) is merely the exterior derivative of (6.18), that is,

$$
\sum_{\beta}\left(d \tau_{\beta} \wedge \theta_{\beta a}+\tau_{\beta} d \theta_{\beta a}\right)-\sum_{i}\left(d \tau_{i \alpha} \wedge \theta_{i}+\tau_{i \alpha} d \theta_{i}\right)=0 .
$$

As for (6.17), we have, by straightforward computations,

$$
\begin{aligned}
d f^{*}\left(\tau \perp \Theta_{\alpha}\right)= & \sum_{i}(-1)^{i-1}\left\{d \tau_{i \alpha}+\sum_{j} \tau_{j a} \theta_{j i}\right\} \wedge \theta_{1} \wedge \cdots \wedge \hat{\theta}_{i} \wedge \cdots \wedge \theta_{n} \\
d \Theta_{\alpha}= & \sum_{\beta, i} \sum_{j \neq 1} \tau_{\beta} \omega_{1} \wedge \cdots \wedge \omega_{j-1} \wedge \omega_{j \beta} \wedge \omega_{j+1} \\
\wedge \cdots \wedge \omega_{i-1} \wedge \omega_{i a} \wedge \omega_{i+1} \wedge \cdots \wedge \omega_{n} & -\sum_{\beta} \bar{R}_{a \beta}{ }^{\tau} \omega_{1} \wedge \cdots \wedge \omega_{n} \\
& -\sum_{\beta, i}(-1)^{i} \tau_{i \beta} \omega_{\beta a} \wedge \omega_{1} \wedge \cdots \wedge \hat{\omega}_{i} \wedge \cdots \wedge \omega_{n} \\
& + \text { terms at least linear in } \omega_{\gamma},
\end{aligned}
$$

where we have set $\bar{R}_{\alpha \beta}=\Sigma_{i} R_{i \alpha_{i} \beta}$. Since $f\left(F^{a}\right)$ is an integral manifold of $I$, we have

$$
f^{*}\left(\omega_{a}\right)=0, \quad f^{*}\left(\omega_{i a}\right)=\sum_{j} b_{i \alpha_{j}} \theta_{j},
$$

where $b_{i \alpha_{j}}=b_{j \alpha_{i}}$ and $\Sigma_{i} b_{i a_{i}}=0$. Using these facts, we have

$$
\begin{aligned}
f^{*}\left(\tau \perp d \Theta_{\alpha}\right)= & \sum_{\beta, i}(-1)^{i-1} \tau_{i \beta} \theta_{\beta \alpha} \wedge \theta_{1} \wedge \cdots \wedge \hat{\theta}_{i} \wedge \cdots \wedge \theta_{n} \\
& -\sum_{\beta}\left(\bar{R}_{\alpha \beta}+\sigma_{\alpha \beta}\right) \tau_{\beta} \theta_{1} \wedge \cdots \wedge \theta_{n},
\end{aligned}
$$

where we have set $\sigma_{\alpha \beta}=\Sigma_{i, j} b_{i \alpha_{j}} b_{i \beta}$.

Therefore, (6.17) can be written as 


$$
\begin{aligned}
\sum_{i}(-1)^{i-1}\left(d \tau_{i a}+\sum_{j} \tau_{j a} \theta_{j i}+\sum_{\beta} \tau_{i \beta} \theta_{\beta a}\right) \wedge \theta_{1} \wedge \cdots \wedge \widehat{\theta}_{i} \\
\wedge \cdots \wedge \theta_{n}-\sum_{\beta}\left(\bar{R}_{\alpha \beta}+\sigma_{\alpha \beta}\right) \tau_{\beta} \theta_{1} \wedge \cdots \wedge \theta_{n}=0 .
\end{aligned}
$$

(6.18) and (6.24) give the equations of variation of $I$. Observe that (6.18) implies the $\tau_{i a}$ components of an $I$-field for which $\tau_{i}=0$ are completely determined once the $r_{a}$ components are given.

Recall that $f\left(F^{a}\right)$ is the bundle space of the bundle of adapted frames over the minimal submanifold $f\left(F^{a}\right)$ of $M$. Let $e=\left(e_{i}, e_{\alpha}\right): W \rightarrow f\left(F^{a}\right)$ be an arbitrary section of the adapted frame bundle over an open set $W$ of $N$. Let $\tau$ be an $I$-field as above. If we put $\tau^{*}=\pi_{*}(\tau)$, then we can write

$$
\tau^{*}(x)=\sum_{a} \tau_{a}(e(x)) e_{\alpha}(x) \stackrel{\text { de f }}{=} \sum_{a} \tau_{a}^{*}(x) e_{\alpha}(x) .
$$

Pulling (6.18) back to $W$,

$$
\sum_{i} \tau_{i a}(e(x)) e^{*}\left(\theta_{i}\right)=d \tau_{a}^{*}+\sum_{\beta} \tau_{\beta}^{*} e^{*}\left(\theta_{\beta a}\right)=\sum_{i} \tau_{a ; i}^{*} e^{*}\left(\theta_{i}\right)
$$

where $\alpha ; i$ here denotes the covariant derivative with respect to the induced normal connection on the submanifold $\pi \circ \bar{f}\left(F^{a}\right)$ in the direction of $e_{i}$. If we write $\bar{R}_{\alpha \beta}=e^{*}\left(\sigma_{\alpha \beta}\right)$, then the pullback of (6.24) after taking $(6.18)$ into account is

$$
\left\{\sum_{i} \tau_{a ; i ; i}^{*}+\sum_{\beta}\left(\bar{R}_{a \beta}+\sigma_{\alpha \beta}\right) \tau_{\beta}^{*}\right\} \cdot e^{*}\left(\theta_{1} \wedge \cdots \wedge \theta_{n}\right)=0
$$

where we have put

$$
d \tau_{\alpha ; i}^{*}+\sum_{j} \tau_{\alpha ; j}^{*} e^{*}\left(\theta_{j i}\right)+\sum_{\beta} \tau_{\beta ; i}^{*} e^{*}\left(\theta_{\beta a}\right)=\sum_{j} \tau_{a ; i ; j}^{*} e^{*}\left(\theta_{j}\right) .
$$

Therefore, the normal vector field $\tau^{*}$ on $\pi \circ \bar{f}\left(F^{a}\right)$ satisfies the following system of of partial differential equations,

$$
\sum_{i} \tau_{a ; i ; i}^{*}+\sum_{\beta}\left(\bar{R}_{\alpha \beta}+\sigma_{\alpha \beta}\right) \tau_{\beta}^{*}=0
$$

which is the so-called Jacobi equation defined on the minimal submanifold $\pi \circ \bar{f}\left(F^{a}\right)$ (see, for example, [4] and [12]). Any normal vector field which satisfies (6.29) is called a Jacobi field. The Jacobi equations were obtained previously in, for example, [4] or [6] by considering the second variations of the $n$-dim volume integral. It is given in the present form in [4].

Proposition 6.2. Let $\tau$ be an I-field on an integral manifold $F^{a}$ of I sucb that $\left\langle\tau, \omega_{i}\right\rangle=0$, then $r^{*}=\pi_{*}(\tau)$ is a Jacobi field on $\pi\left(F^{a}\right)$. Conversely, for any Jacobi field $\tau^{*}$ on $\pi\left(F^{a}\right)$, there exists an I-field $\tau$ on $F^{a}$ such that $\pi_{*}(\tau)=\tau^{*}$.

Proof. We have already proved the first half of the proposition. If $\tau^{*}=$ $\Sigma_{a} \tau_{a}^{*} e_{\alpha}$ is a Jacobi field on $\pi\left(F^{a}\right)$, by taking different sections of the adapted 
frame bundle $F^{a}$ over $\pi\left(F^{a}\right)$ we can define the $\tau_{a}$ components of an I-field $\tau$ on $F^{a}$ by $(6.25)$ and hence also its $\tau_{i a}$ components by (6.26). If we put $0=$ $\left\langle\tau, \omega_{i}\right\rangle$ and choose the components $\tau_{\alpha \beta}=\left\langle\tau, \omega_{\alpha \beta}\right\rangle, \tau_{i j}=\left\langle\tau, \omega_{i j}\right\rangle$ arbitrarily, then we have an $I$-field $r$ on $F^{a}$ such that $\pi_{*}(r)=r^{*}$. Q.E.D.

Theorem 6.2. Let $M$ be a Riemannian manifold of dimension $n+p$ and $N$ be an n-dim minimal submanifold of $M$. Let $\mathrm{v}: N \rightarrow T(M)$ be a Jacobi field defined on $N$. Under the assumption of real analyticity, for any point $m^{\circ} \in N$ there exists a one-parameter family of minimal submanifolds ${ }^{t} N(t \in(-\epsilon, \epsilon)$ and $\epsilon>0)$ in a neighborbood 60 of $m^{\circ}$ in $M$, such that ${ }^{0} N=6 \cap \cap N$ and the deformation vector of ${ }^{t} N$ on ${ }^{0} N$ coincides with the restriction of $\mathrm{v}$ to ${ }^{0} N$.

Proof. The bundle space of the bundle of adapted frames $F^{a}(N)$ of $N$ is an integral manifold of $I$. In fact, as noted earlier, it is a general solution of $I$. By Proposition 6.2 there exists an $I$-field $\tau$ on $F^{a}(N)$ such that $\pi_{*}(\tau)=\mathbf{v}$ and $0=$ $\left\langle\tau, \omega_{i}\right\rangle=\left\langle\tau, \omega_{\alpha \beta}\right\rangle=\left\langle\tau, \omega_{i j}\right\rangle$. Then $\tau$ is an $I$-field which satisfies the conditions of the Main Theorem. Since a one-parameter family of integral manifolds of $I$ on which (6.12) holds is mapped under $\pi$ onto a one-parameter family of minimal submanifolds, Theorem 6.2 follows now directly from the Main Theorem Q.E.D.

\section{BIBLIOGRAPHY}

1. R. L. Bishop and R. J. Crittenden, Geometry of manifolds, Pure and Appl. Math., vol. 15, Academic Press, New York, 1964. MR 29 \#6401.

2. G. A. Bliss, Lectures on the calculus of variations, Phoenix Science Series, 1963.

3. E. Cartan, Les systèmes différentiels extérieurs et leurs applications géométrique, Actualités Sci. Induist., no. 994, Hermann, Paris, 1945. MR 7, 520.

4. S. S. Chern, Minimal submanifolds in a Riemannian manifold, University of Kansas, Department of Mathematics Technical Report 19 (New Series), Univ. of Kansas, Lawrence, Kan., 1968. MR $40 \# 1899$.

5. R. Hermann, E. Cartan's geome tric theory of partial differential equations, Advances in Math. 1 (1965), fasc. 3, 265-317. MR 35 \#520.

6. - The second variation for minimal submanifolds, J. Math. Mech. 16 (1966), 473-491. MR 34 \#8348.

7. E. Kähler, Einführung in dte Theorie der System von Differentialgleichungen, Chelsea, New York, 1949.

8. S. Kobayashi and K. Nomizu, Foundations of differential geometry. Vol. 1, Interscience, New York, 1963. MR 27 \#2945.

9. M. Kuranishi, Lectures on exterior differential systems, Tata Institute of Fundamental Research, Bombay, 1962.

10. A. B. Poritz, A generalization of parallelism in Riemannian geometry, the $C^{\omega}$ case, Trans. Amer. Math. Soc. 152 (1970), 461-494. MR 42 \#3710.

11. Y. Matsushima, On a theorem concerning the prolongation of a differential system, Nagoya Math. J. 6 (1953), 1-16. MR 15, 428.

12. J. Simons, Minimal varieties in riemannian manifolds, Ann. of Math. (2) 88 (1968), 62-105. MR 38 \#1617.

DEPARTMENT OF MATHEMATICS, BROWN UNIVERSITY, PROVIDENCE, RHODE ISLAND 02912

Current address: Department of Mathematics, Purdue University, Lafayette, Indiana 47907 